\title{
Gradhiva
}

GRADHIV

Revue d'anthropologie et d'histoire des arts

$20 \mid 2014$

Création fiction

\section{Les trois anges de Séraphine de Senlis}

The three angels of Séraphine de Senlis

\section{Giordana Charuty}

\section{(e) OpenEdition}

Journals

Édition électronique

URL : https://journals.openedition.org/gradhiva/2856

DOI : 10.4000/gradhiva.2856

ISSN : 1760-849X

\section{Éditeur}

Musée du quai Branly Jacques Chirac

\section{Édition imprimée}

Date de publication : 1 octobre 2014

Pagination : 108-137

ISBN : 978-2-35744-074-6

ISSN : 0764-8928

\section{Référence électronique}

Giordana Charuty, «Les trois anges de Séraphine de Senlis », Gradhiva [En ligne], 20 | 2014, mis en ligne le 01 octobre 2017, consulté le 21 septembre 2021. URL : http://journals.openedition.org/ gradhiva/2856 ; DOI : https://doi.org/10.4000/gradhiva.2856

(c) musée du quai Branly 


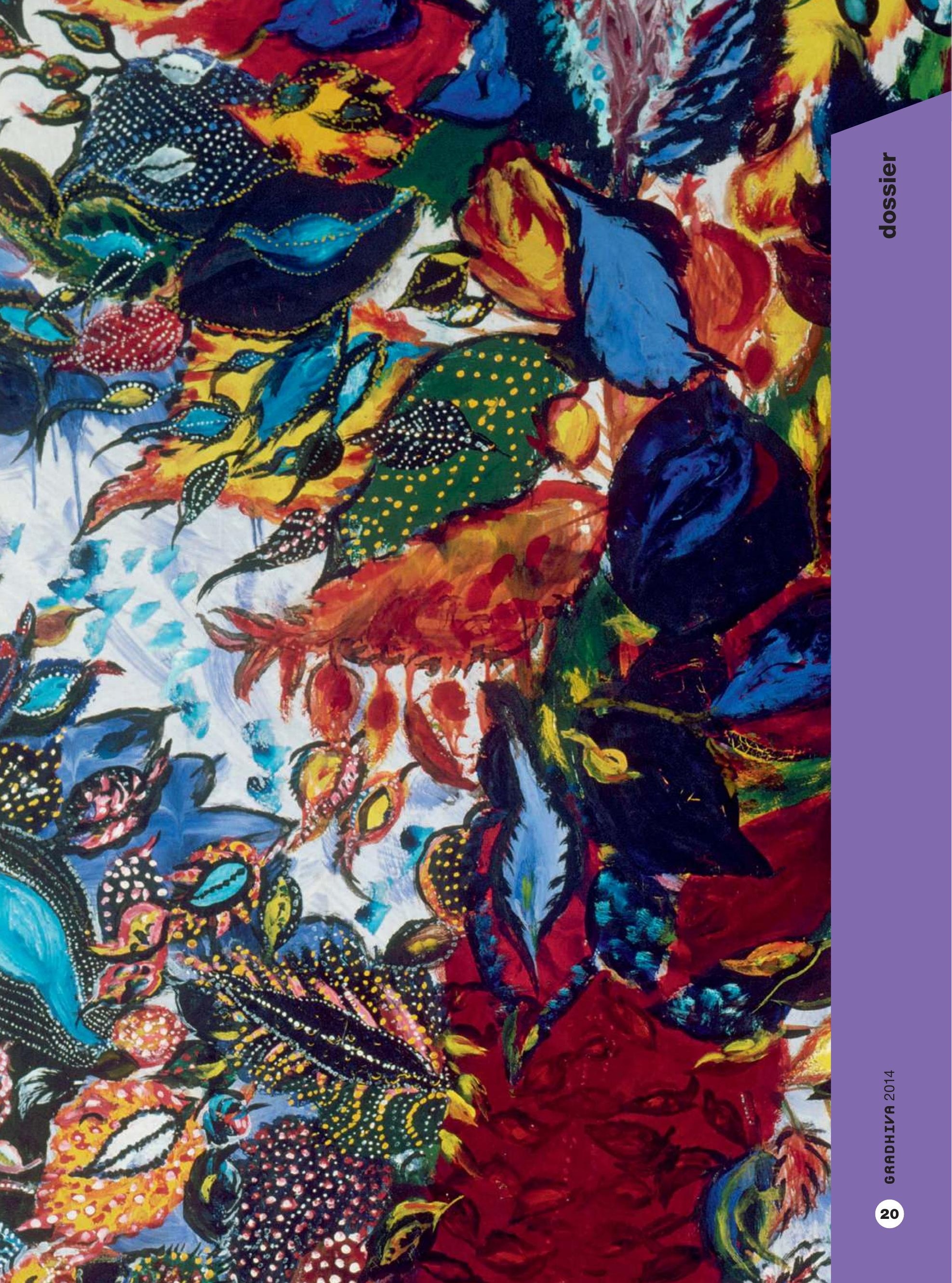





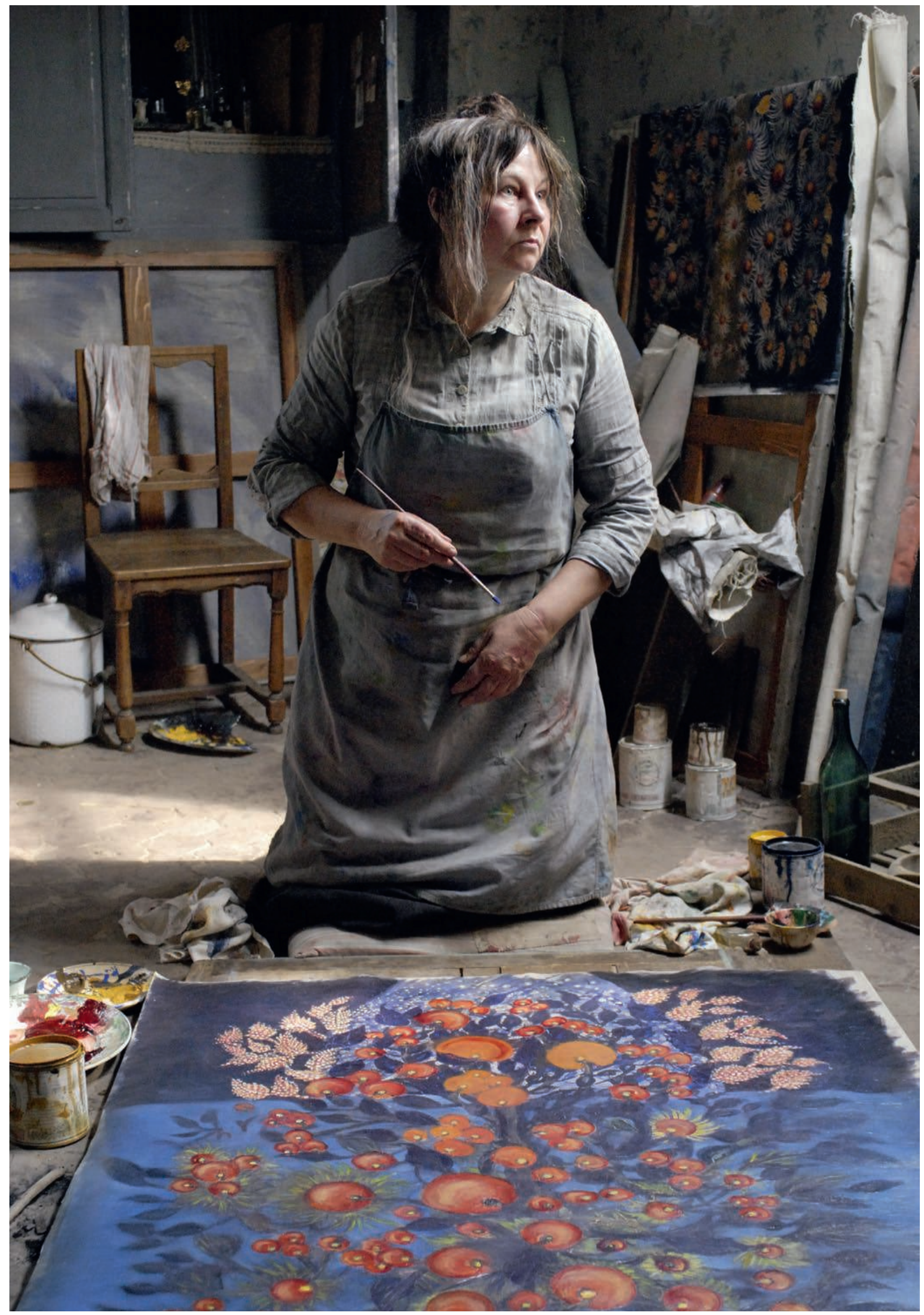


«La peinture n'a pas la clé du cinéma, mais elle fait partie, avec toute son histoire et ses symboles et ses conventions, du mystère de l'interprétation en cinéma", rappelle Jacques Aumont dans l'avant-propos à une réédition de L'CEil interminable (2007 [1989]: 15). L'étude pionnière, parue en 1989, contraste les deux catégories d'images pour qualifier le régime de figuration propre à un langage plastique à l'identité incertaine: une «machinerie psychique et sociale», affirme l'auteur, qui soude un groupe social autour de représentations partagées et offre à chacun un répertoire de formes pour assumer une position dans le monde. Après bien d'autres, le film dont il sera question ici instaure une relation en miroir entre ces deux arts visuels. II donne à voir l'acte de peindre pour énoncer quelque chose sur l'activité de création. Une fiction, donc, qui se donne pour telle puisqu'il y a narration, jeu d'acteur et mise en scène. Mais les principaux personnages renvoient à des individualités qui ont réellement existé, le réalisateur a tourné sur leurs lieux de vie et s'est appuyé sur un important travail documentaire. Comment, dans un récit qui brouille les frontières entre le fictif et le réel pour affirmer une validité référentielle, l'image de cinéma est-elle appelée à rendre compte de l'apparition de l'image de peinture?

\section{Ceci n'est pas une biographie}

Malgré un budget modeste, la sortie en salles de Séraphine, en octobre 2008, a été soigneusement préparée comme un événement culturel à plusieurs facettes pour faire se rejoindre l'œuvre et la vie d'une artiste autodidacte, appréciée de quelques amateurs de peinture dite naïve mais, jusqu'alors, méconnue du grand public. Tandis que le film de Martin Provost lui restitue un visage et une présence charnelle, le musée Maillol donne à voir dix-huit tableaux de celle qui s'était, elle-même, proclamée «peintre sans rivale». La librairie n'est pas en reste: catalogue et biographies paraissent qui opèrent la fusion de l'artiste et de l'actrice, Yolande Moreau, en mêlant plans du film, photographies et reproductions de tableaux ${ }^{\mathbf{1}}$. Dans le même temps, on réédite deux des portraits - Séraphine de Senlis et Henri Rousseau - que le critique d'art Wilhelm Uhde avait rassemblés, en 1949, dans la même catégorie de «maîtres primitifs ${ }^{2}$ ". Avec de très nombreux dossiers de presse, des dossiers pédagogiques, des débats dans les établissements scolaires, l'œuvre qu'un réalisateur peu connu a consacrée à une artiste elle-même ignorée $a$, de fait, suscité une émotion esthétique collective. Elle lui vaut l'obtention, en février 2009, d'une rare consécration par l'académie des Césars $^{3}$. Comment un cinéaste en vient-il à s'intéresser à une artiste en marge de l'histoire de l'art et à imposer sa reconnaissance à une aussi grande diversité de publics?

Acteur de théâtre, écrivain, cinéaste: Provost est un artiste «pluriel», diraient les sociologues ${ }^{4}$. Un rêve où il se voit «le ventre plein de manuscrits 5 " l'a déterminé à ne plus prêter son corps et sa voix aux textes des autres. Quant au choix de Séraphine, ce n'est pas sa peinture mais son histoire qui a d'abord retenu son attention et l'a incité à entreprendre sa propre enquête à travers les textes, les lieux et les sources disponibles. Or, parti sur les traces d'une domestique artiste autodidacte, il entrevoit une autre vie qui va le retenir tout autant: celle de son découvreur et mécène allemand Uhde. Faire revivre cette rencontre improbable entre deux marginalités motive le parti pris narratif: «Je ne souhaitais pas faire une
1. Chez Albin Michel, Séraphine, de la peinture à la folie est une version remaniée d'une biographie parue vingt ans auparavant dans une collection dirigée par Georges Duby (Vircondelet 1986 et 2008). L'auteur a, d'abord, retenu le nom de Séraphine de Senlis, donné par le collectionneur et marchand d'art Wilhelm Uhde lors de la première exposition parisienne exclusivement consacrée, en 1945, à l'artiste morte peu avant à l'asile de Clermont-de-l'Oise.

La nouvelle version met en avant le basculement dans la folie et l'internement d'une "autre Camille Claudel ».

Tout comme le catalogue de l'exposition, la "vie rêvée" de Séraphine, éditée par les éditions Phébus, associe l'artiste et l'actrice (Cloarec 2008). L'auteure a été consultée par le cinéaste pour avoir, au milieu des années 1980, consacré à Séraphine une thèse de psychologie clinique.

2. Uhde 2008. Les éditions du Linteau ont auparavant publié une précieuse traduction française des mémoires du critique allemand (Uhde 2002).

3. Sept Césars pour le film, le scénario, l'actrice, les costumes, la photographie la musique, le décor. Les vainqueurs précédents d'un tel palmarès sont Alain Resnais, Louis Malle et Roman Polanski.
4. Outre deux longs métrages, il a été acteur de théâtre durant dix ans, dont six comme pensionnaire de la Comédie Française, et a publié plusieurs romans.

5. Sous les traits de Zouk, personnage féminin de Claire Bretécher, voir : Lisa Vignoli, "Martin Provost, séraphique», Libération, 2 mai 2011.

\section{ci-contre}

fig. 1

Yolande Moreau interprétant Séraphine Louis dans le film Séraphine de Martin Provost, 2008. Production TS Productions. Distribution Diaphana. Photo (C) Michaël Crotto. 
6. Interview de Yolande Moreau et Martin Provost, lepetitjournal.com/sortir/ cinema-madrid/40462, 5 mai 2012.

7. Outre l'historicité du lien entre ces deux arts visuels, on interroge les manières dont le cinéma réfléchit à la peinture, déplace ses questions conceptuelles, pour penser son propre régime d'image. Cette question traverse toute la réflexion d'André Bazin. Après l'étude de Jacques Aumont (1989), la meilleure synthèse est celle d'Antonio Costa (1991 et 2002, avec une importante note bibliographique), suivie par plusieurs livraisons de revues: "Peinture et cinéma. Picturalité de l'image filmée, de la toile à l'écran ", Ligeia, nos 77 à 80 (2008), et un état des lieux qui souligne le tournant esthétique des derniers travaux, au détriment d'enquêtes historiques comme celles sur l'importance des débuts du cinéma pour les avant-gardes artistiques qui entendent rejeter les conventions de la représentation: Albera 2008.
8. Le programme «Filmer la création artistique ", conduit à l'université de Rennes, présente un corpus, identifie les codes du genre fixé dans les années 1950 - par John Huston, avec Moulin Rouge (1952) et par Vincente Minnelli, avec La Vie passionnée de Van Gogh (1956) - ainsi que les formes possibles de transgression. Parmi la soixantaine de films répertoriés, on trouve quelques grands peintres de l'Ancien Régime (Rembrandt Le Greco, Goya, Le Caravage) et, surtout, de nombreuses incarnations de l'artiste moderne (Van Gogh, Klimt, Munch, Picasso, Gauguin, etc.), mais seulement deux femmes: Frida Kahlo et Séraphine Louis, à laquelle Patrick Louguet consacre une étude décevante dans la mesure où on ne peut caractériser le «régime de fiction cinématographique " si on l'isole des autres narrations de Séraphine (Thivat 2011).

9. On peut reconstituer l'ensemble de ces textes à partir de la minute du jugement du tribunal de grande instance de Paris rendu le 26 novembre 2010. En réponse à l'accusation de "contrefaçon des droits d'auteur " portée par Alain Vircondelet et les éditions Albin Michel, Provost identifie toutes les sources, citées ou non citées, que le biographe a lui-même utilisées. biographie de Séraphine: je voulais mettre en lumière le processus créateur. Montrer comment, à un moment ou un autre, une rencontre est indispensable à l'artiste ${ }^{6}$. "

Cette déclaration doit s'entendre comme le rejet d'un genre cinématographique, le biopic, qui au-delà des artistes inclut désormais toutes les figures historiques; mais ce sont bien les vies de peintres, présentes depuis le Rembrandt d'Alexandre Korda (1936) dans toute l'histoire du cinéma, qui ont imposé le genre de la biographie filmée. L'abondante réflexion critique et théorique produite depuis le début des années 1980 sur les rapports entre cinéma et peinture est d'actualité dans les revues d'histoire culturelle du cinéma au moment même de la sortie de Séraphine ${ }^{7}$. Les études qui interrogent ces vies où le grand peintre figure l'artiste par excellence sont, néanmoins, d'un intérêt inégal ${ }^{8}$. À partir de l'idée banale que ces dernières construisent une légende, on s'emploie à comparer les portraits filmés d'un même peintre pour contraster l'usage des données historiques, l'interprétation que chaque cinéaste propose des rapports au pouvoir politique, la diversité des contenus idéologiques qui nourrissent le mythe de l'artiste maudit. On interroge l'existence de traditions nationales ou bien l'on s'efforce de caractériser la biographie totale, exemplairement incarnée par l'Edvard Munch de Peter Watkins (1974). Enfin, on décrit l'imaginaire du peintre au travail en suivant quelques topiques: par exemple, la nuit des ateliers, thème d'autant plus surprenant que le principe de peindre en lumière diurne est toujours respecté au xx siècle (Thivat 2011). II nous faut donc reprendre la question: comment met-on à distance un genre et ses codes narratifs pour poursuivre «en cinéma» une interrogation sur l'acte de création «en peinture»?

Le cinéaste a lui-même écrit le scénario avec le dialoguiste Marc Abdelnour en usant d'une méthode largement pratiquée par ses prédécesseurs, traiter comme documentation historique un ensemble hétérogène de textes: la première biographie publiée en 1986 à partir d'une thèse en histoire de l'art, des thèses de médecine inédites, des portraits de critiques d'art et de témoins, des catalogues d'exposition, le récit autobiographique de Uhde ${ }^{9}$. Laissons de côté la manière dont l'œuvre filmée a été convoquée dans l'espace judiciaire pour une expertise quant au statut de cette production écrite et lisons-la à notre tour en ethnographe, en suspendant provisoirement les catégories de genre textuel. Une première constatation s'impose alors: sous une apparente répétitivité, ces textes ne construisent pas la même «Séraphine». Elle motive une décision de méthode: les traiter ni comme des sources documentaires pour mesurer la validité référentielle de la création filmique, ni comme des récits fictionnels engageant la question de l'authenticité à partir du lien entre l'œuvre et le cinéaste; considérer, au contraire, récits écrits et filmé comme autant de narrations tributaires de l'expérience de chaque enquêteur ou témoin, de son domaine d'expertise, de ses ressources expressives et de ses engagements culturels. Ce faisant, les mémoires de Uhde et le portrait qu'il nous a laissé de l'unique femme admise au rang de «maître primitif» nous sont, progressivement, apparus comme les meilleurs guides pour «lire» le récit filmé de Provost. Non parce que celui-ci en interprète, assez fidèlement, des épisodes mais parce qu'entrer dans l'univers du collectionneur nous fait mieux voir les 


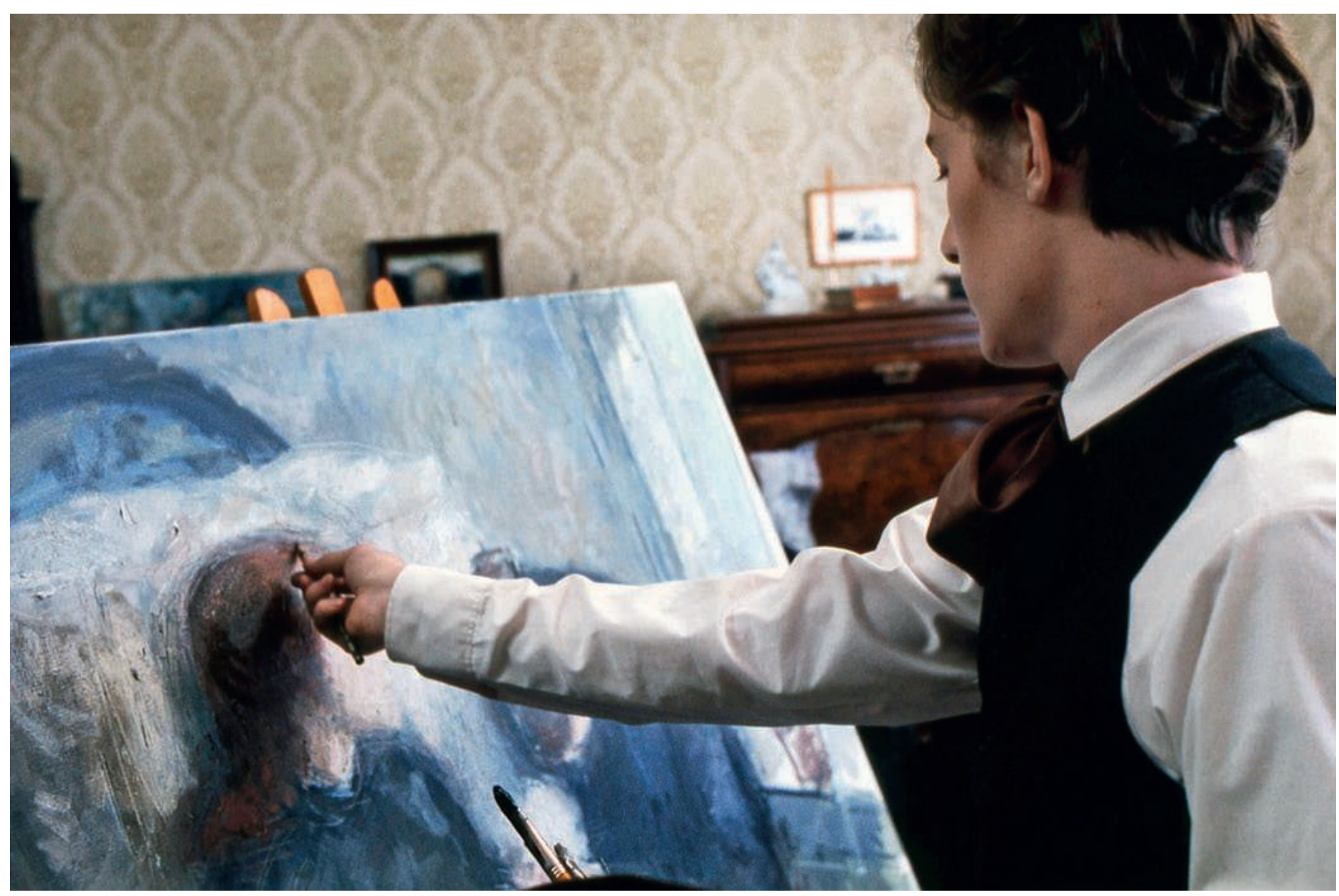

fig. 2

Geir Westby dans le film

Edvard Munch de

Peter Watkins, 1973.

Avec l'aimable autorisation

de Peter Watkins et

Doriane Films. 
10. Elles lui valent un succès d'estime en Allemagne comme auprès des critiques d'art et des artistes allemands qui vivent en Italie. solutions visuelles inventées par le cinéaste pour installer le spectateur en témoin de ce dont «Séraphine» est le nom: à savoir les divers registres d'une lutte sans répit pour conquérir une puissance créatrice, en reconnaissant le tableau pour destin.

\section{L'esprit gothique}

Une question sous-tend les mémoires qu'âgé d'un peu plus de 60 ans Uhde écrit à Paris durant l'année 1936, qui lui valent d'être déchu de la nationalité allemande après leur publication en Suisse en 1938 et qui n'ont été traduits en français qu'en 2002, sous le titre De Bismarck à Picasso: comment met-on le tableau au centre de sa vie? Cela commence à Posen où, tout jeune enfant, Uhde circule entre les diverses demeures d'une riche et vaste famille prussienne, mi-catholique, mi-protestante, et peut très concrètement éprouver la différence religieuse qui marque le rapport aux images. Par opposition à la lignée paternelle d'austères pasteurs protestants ne possédant ni bibelots, ni tableaux, la famille maternelle n'a pas seulement un rapport hédoniste aux objets décoratifs. Une tante, lui montrant un tableau du seul artiste de la famille, l'avertit: " "Regarde bien, mon garçon, ce tableau te suivra des yeux partout où tu iras." J'y fis bien attention et pus constater à ma grande surprise qu'elle avait raison. » (Uhde 2002: 19) Cette première expérience d'emprise d'un portrait peint opère une substitution remarquable. Un ancêtre en image vient prendre la place des êtres auxquels son christianisme réformé prête une omniscience menaçante - Dieu et l'ange gardien -, comme en témoigne la déclaration d'incroyance que le jeune Wilhelm adresse, dans le même temps, aux «faits réels»: «Un fait établi, c'était notamment le bon Dieu dont la présence continue et obstinée me gênait beaucoup. Pour m'y opposer, je restais agenouillé pendant des heures devant un poêle de faïence blanche à qui je clamais, au grand dam de ma mère: "Je t'adore, ô mon idole." " (Ibid. : 24) La genèse, à partir de Nicolas de Cuse, du motif de l'image de Dieu ou du Christ, dont le regard suit partout le spectateur, est retracée par Carlo Ginzburg (2013:84-85). Celle-ci s'accompagne aussi de la fascination pour la profusion d'images colorées qui distingue l'univers catholique de la mère Nicólai, une servante polonaise de sa grand-mère: des images de la Vierge et des saints, des vases en verre argenté emplis de «fleurs artificielles aux couleurs incroyables» (ibid. : 23).

Le deuxième ébranlement esthétique a lieu lorsque, ayant terminé des études de droit, Uhde échappe à une carrière de petit magistrat dans une ville de la pluvieuse province prussienne en obtenant, à titre de cure médicale, de séjourner en Italie. À Florence, puis à Rome, il découvre une esthétique: I'harmonie du paysage, de l'homme et de l'art qu'ont su réaliser les primitifs italiens; un milieu social: celui des jeunes historiens de l'art allemands; et une liberté de mœurs qui l'autorise à reconnaître son homosexualité: les trois registres de ce qu'il appelle une «révélation ». Entre deux séjours italiens, il renonce définitivement à toute carrière juridique, publie des Lettres florentines sur la civilisation allemande, une sorte de manifeste sur les idéaux que devrait se donner la culture allemande ${ }^{\mathbf{1 0}}$, et commence des études d'histoire de l'art à Munich, puis à Breslau où il fréquente les séminaires de Richard Muther en compagnie d'Erich Klossowski. Mais comment «vivre les tableaux» par le cœur plutôt que par la pensée et les livres? 
C'est à Paris que Unde va, enfin, découvrir cette singulière expérience sensible. Son ami Klossowski l'a précédé de quelques mois lorsqu'il arrive, à son tour, en 1904. Il a 29 ans. Très vite, il devient une figure centrale de cette colonie allemande du Dôme qui commence à se former à Montparnasse ${ }^{\mathbf{1 1}}$ où, dans deux petites salles obscures d'un bureau de tabac, se retrouvent artistes, historiens et critiques d'art, marchands et modèles en provenance de l'Europe nordique et centrale. Les visites assidues aux galeries, salons, foires, ateliers d'artistes et de collectionneurs, et les interminables discussions nocturnes vont rendre le tableau vital à un double titre, matériel et psychique. «Le sens de la qualité s'affirmait peu à peu par la fréquentation quotidienne de tableaux que l'on désirait passionnément avant de les posséder, de les tenir tous les jours entre les mains, de les poser ici et là et de les exposer à tel ou tel éclairage, que l'on défendait contre tous les doutes et les critiques, et qu'enfin l'on ne pouvait se décider à revendre avant que la misère nous y eût contraint ou que l'on eût trouvé un autre tableau que l'on pensait aimer davantage. " (Ibid.: 125) La collection que Uhde réunit en dix ans est remarquable: dix-sept Braque, douze Picasso, cinq Dufy, un Léger et cinq Rousseau, plus des aquarelles et des dessins ${ }^{\mathbf{1 2}}$. Les Picasso et les Braque qu'il achète alors relèvent de cette expérimentation commune aux deux peintres que, rétrospectivement, la critique a qualifiée de cubisme analytique. Une «prolifération des géométries", suggère Philippe Dagen, qui fait disparaître le sujet du tableau par fragmentation des plans, glissement des volumes les uns sur les autres, abolition de la distinction entre espace intérieur et extérieur. En somme, un moment d'abstraction aussitôt suivi, note l'historien de l'art, de la réintroduction de l'objet à partir d'autres manières de le désigner: les lettres dans le tableau, les papiers collés, le contre-emploi des matériaux du commerce ${ }^{13}$.

Comme en écho à cette inquiétude face à la disparition de l'objet, Unde s'engage, parallèlement, dans la reconnaissance d'une peinture qui préserve à sa façon la figuration. C'est la mère de Robert Delaunay qui le conduit dans la petite chambre où celui que l'on appelle le Douanier Rousseau est en train de peindre La Charmeuse de serpents. Séduit par l'étrangeté de ce vieil homme en lequel il perçoit à la fois un enfant et un grand artiste, Unde observe attentivement cette manière somptueuse de peindre qui retrouve certaines des solutions de ses chers primitifs toscans pour perturber la représentation ${ }^{\mathbf{1 4}}$. C'est décidé: ce sera le premier artiste exposé en 1908 «chez Ulk», au 73, rue Notre-Dame-des-Champs, une galerie minuscule qu'un marchand de meubles du faubourg Saint-Antoine met à sa disposition ${ }^{15}$. Un an auparavant, son ami Daniel-Henry Kahnweiler a ouvert une galerie où il expose exclusivement Pablo Picasso, Georges Braque et André Derain, mais il considère la peinture du Douanier Rousseau - «fleur miraculeuse de l'art populaire» - comme la seule à pouvoir, en toute rigueur, être qualifiée, avant 1910, d'«art primitif » (Kahnweiler 1990 [1946]: 199). Le livre de souvenirs et d'hommage que Uhde publie en 1911, après la mort du peintre, invente un style de biographie d'artiste qui anticipe celui de Hans Prinzhorn et sera systématisé pour les créateurs d'art brut: une présentation où la forme de vie participe de l'œuvre à reconnaître. Cette différence de traitement critique ne signifie pas une hiérarchie des valeurs picturales: Picasso, Braque, le Douanier Rousseau, Marie Laurencin sai-
11. Souvent évoquée dans les écrits de peintre et les récits biographiques, elle a fait l'objet d'une étude détaillée: Gautherie-Kampka 1995.

12. Bernard Marrey en a établi la liste à partir du catalogue de leur vente à l'hôtel Drouot le 30 mai 1921, en tant que biens "ennemis" séquestrés. II note que le directeur des Beaux-Arts n'a pas jugé bon d'exercer le droit de préemption de l'État pour faire entrer cette collection dans les musées français. En 1921 et 1923, c'est à son tour le séquestre Kahnweiler qui sera vendu aux enchères (in Unde 2002 : 287-296).

13. Philippe Dagen (2008: 91-170) souligne les réticences de Georges Braque et de Pablo Picasso à être qualifiés de «cubistes" dans ce moment de construction «jusqu'à l'émiettement ", illustré aussi bien par le Portrait de Daniel-Henry Kahnweiler (1910) que par le Portrait de W. Unde (1910), qui précède l'invention de nouveaux codes. DanielHenry Kahnweiler (1990 [1946] : 181-194) mettait également au centre de la naissance du cubisme le problème du volume, et non l'application d'une géométrie scientifique.

14. La disproportion et le principe de «métonymie visuelle» (Dagen 2008: 106-107)

15. La distraction fut fatale à cette première exposition exclusivement consacrée à Henri Rousseau:

"Malheureusement, j'avais oublié d'indiquer l'adresse exacte de cette petite galerie inconnue sur les cartons d'invitation, si bien qu'il n'y eut personne. " (Uhde 2002: 148) 
16. À savoir exprimer une subjectivité et rejoindre «la vaste conscience universelle du temps " (Uhde 2002: 155) Robert Goldwater s'appuie sur une lecture attentive de Unde pour caractériser la manière « retardataire» d'Henri Rousseau (Goldwater 1986 [1938] : ch. VI).

17. Uhde 2002: 162. Dans une étude bien informée qui entend différencier le légendaire des faits historiquement attestés, Hans Korner et Manja Wilkens ont rassemblé d'autres témoignages qui situent la découverte du premier tableau tour à tour chez un photographe, un cordonnier, un tailleur, un antiquaire (2009: 46).

18. Son livre Picasso et la tradition française. Notes sur la peinture actuelle ne trouvera pas d'éditeur en Allemagne. II sera publié à Paris par les éditions Les Quatre Chemins qui éditent, avec le critique WaldemarGeorge, la revue d'avantgarde Formes à laquelle collabore Unde.

\section{ci-contre}

fig. 3

Couverture de Cinq maitres primitifs de Wilhelm Unde, Paris, Librairie Palmes,

Philippe Daudy éditeur, 1949

Collection particulière. sissent, chacun à leur façon, l'essentiel "sous sa forme plastique primitive", donnant à cette collection la valeur d'une «profession de foi ${ }^{16}$ ».

C'est dans ce contexte que fait, une première fois, irruption Séraphine sur le mode d'une rencontre de hasard que le collectionneur a fixée dans un bref récit souvent repris, décliné en diverses variantes, et que le cinéaste va transposer en lui donnant une plus longue épaisseur temporelle:

Mais quoi que je fisse, où que j'allasse, je ne pouvais plus échapper au «tableau» qui était devenu mon destin et emplissait ma vie. Un jour, je trouvai posée sur une chaise, chez des bourgeois, une nature morte qui me coupa singulièrement le souffle. C'étaient des fruits modelés dans une singulière pâte consistante, au lieu d'être seulement effleurés: des pommes, paraît-il, mais elles étaient faites de beauté et devenues réalité. Le jeune Cézanne aurait pu les peindre. Je demandai qui était l'artiste et l'on me répondit: Séraphine. Comme ce nom ne me disait rien, on me raconta que c'était ma femme de ménage, une vieille dame qui faisait ma chambre depuis quelques jours et à qui je n'avais pas prêté attention. J'achetai ce tableau ainsi que tous ceux que Séraphine avait peints au fil des ans et m'avait apportés, ravie de mon intérêt. J'en montrai quelques-uns à mes amis à Paris, en leur disant qu'ils avaient été peints par une nonne. Mes amis furent aussi surpris et enchantés que moi, et l'un d'entre eux s'écria: «À quoi bon continuer à peindre si une femme inculte peut réaliser des œuvres aussi puissantes ${ }^{17}$ ?"

Cette première rencontre, interrompue par la guerre et la séquestration de la collection du marchand, n'occupe qu'un paragraphe dans le récit autobiographique. Les retrouvailles après guerre sont plus détaillées. À son retour à Paris, en 1924, les artistes que Uhde défendait dix ans auparavant lui sont devenus financièrement inaccessibles. Il se met en quête de peintres "primitifs", au sens que le qualificatif a pris avec Henri Rousseau, tout en se faisant le passeur de Picasso auprès du public allemand ${ }^{18}$. Alors qu'il s'est installé à Chantilly avec sa sœur et son ami, le peintre Helmut Kolle, l'annonce dans le journal local d'une exposition à l'hôtel de ville de Senlis réveille en lui le souvenir de Séraphine Louis. Nous sommes en 1927. À l'écart d'un art provincial aussi étriqué que le monde social d'où il émane, trois grands tableaux «d'une puissance saisissante» s'emparent du regard du collectionneur:

un bouquet de lilas dans un vase noir, un cerisier, deux pieds de vigne chargés, l'un de raisins noirs, l'autre de blancs. Et tandis que je contemplais tranquillement ces toiles de Séraphine, je crus tout à coup entendre s'éveiller et carillonner dans le lointain des cloches depuis longtemps muettes. (Uhde 2002: 242)

Une expérience cénesthésique qui rappelle celle qui accompagne la quête de l'«heure de Moscou" dans les premiers tableaux peints de mémoire à Munich par Wassily Kandinsky (Kandinsky 1974: 91-92).

À Paris, la revue d'avant-garde Formes, financée par le collectionneur Shigeratô Fukushima et dirigée par le très influent critique d'art Waldemar- 


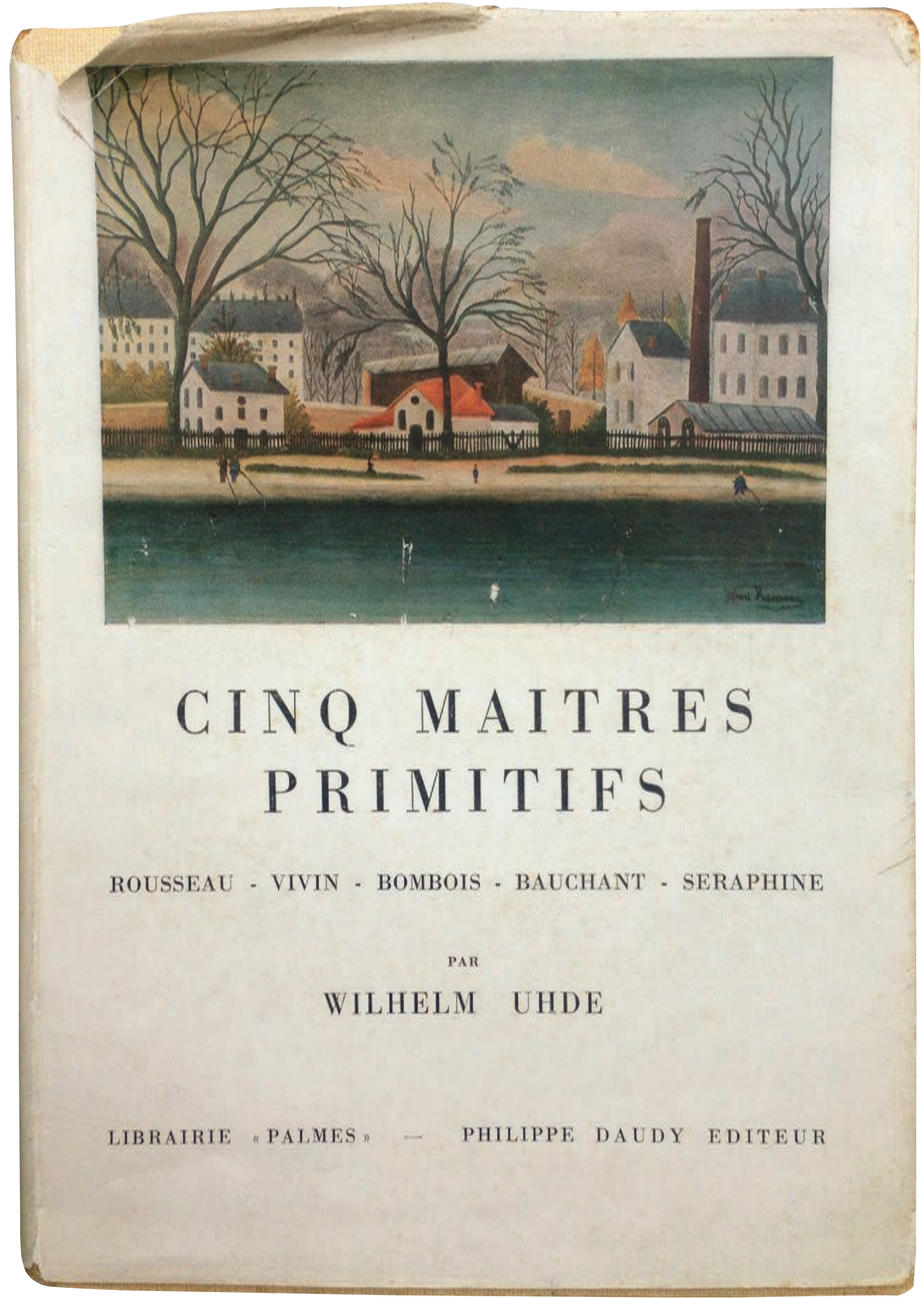




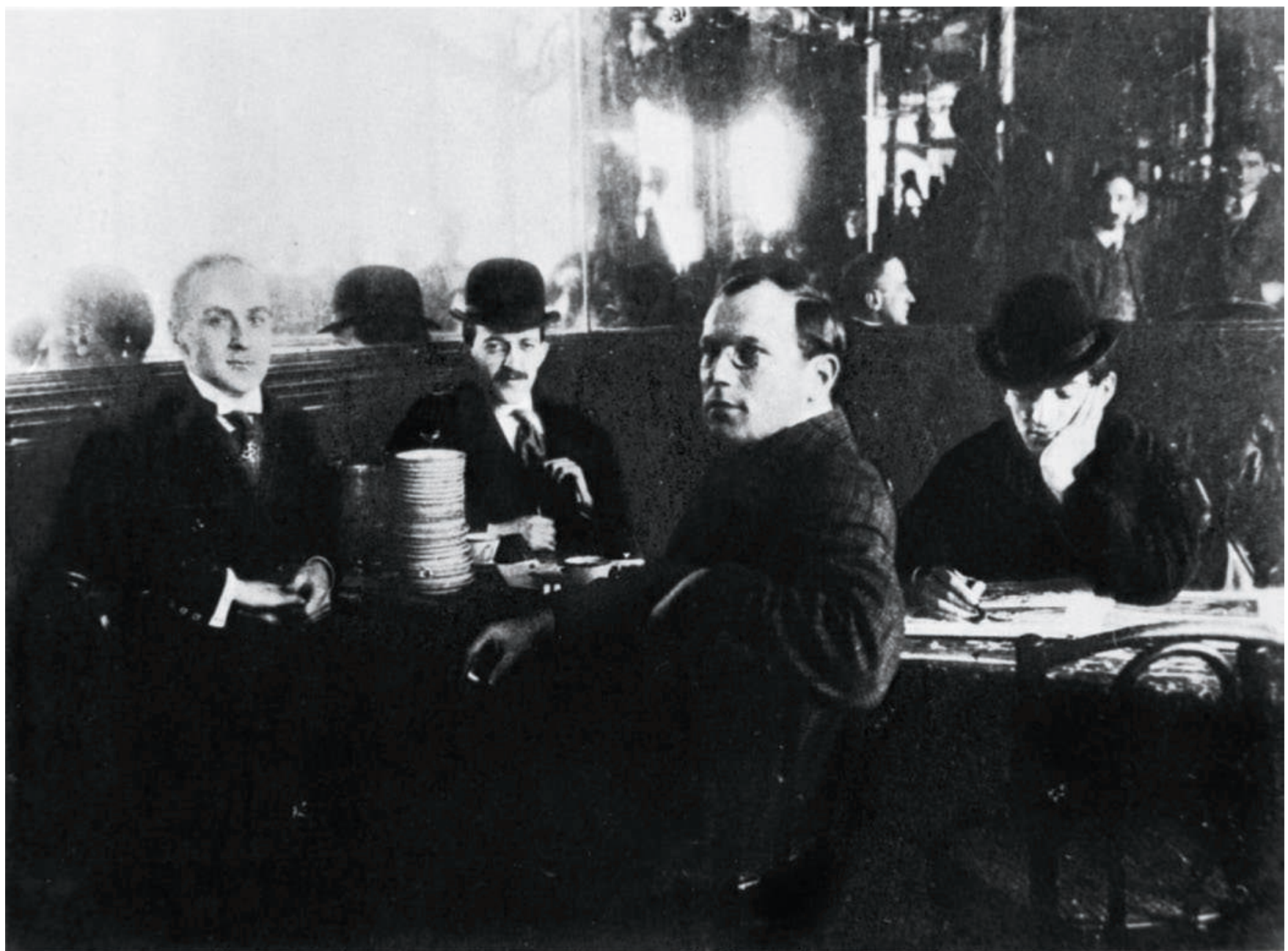

H'u cafe'du Doine en 1908: Aa ford Wuthelm Uhde, Bondy et Parcin; au hremei nlan. Rudell Resu.

fig. 4

De gauche à droite

Wilhelm Unde, Walter Bondy,

Rudolf Levy et Jules Pascin,

attablés au Café du Dôme,

108 boulevard Montparnasse,

1908. Photographe anonyme. 
George, a d'emblée pris parti pour ces peintres sans formation académique - Camille Bombois, André Bauchant - qui côtoient, dans ses pages, Matisse, Picasso, Derain. Le tout jeune Joseph Delteil et le galeriste Henry Bing ripostent avec vigueur à la disqualification qu'André Lhote formule à l'égard de cet art populaire dans la Nouvelle Revue française et à la suspicion jetée sur Uhde, animateur d'un «étrange marché». Celui-ci répond par une «défense de l'art populaire» dans la revue magazine Jazz, tandis que Waldemar-George prend lui-même la défense de Bauchant, en lequel il reconnaît «une vieille tradition populaire [qui] rejoint le pur folklore français, alimenté par l'art gallo-romain, cette genèse, cette matrice initiale ${ }^{19}$ ». Sous les auspices de Uhde, Séraphine, qui vient d'être exposée en 1928 à la galerie des Quatre-Chemins, fait son entrée dans la prestigieuse revue avec deux tableaux, avant de rejoindre ses pairs, les «primitifs modernes », durant l'été 1932 à la galerie Bernheim-Jeune 20.

Après la Seconde Guerre mondiale, le portrait publié en 1949 dans Cinq Maîtres primitifs associe durablement «Séraphine de Senlis» à cet ensemble de peintres - Maurice Utrillo, le Douanier Rousseau, Louis Vivin, Bombois, Bauchant - d'un pôle opposé à la perte de la figuration qui a croisé, avant guerre, un plus vaste débat sur l'art populaire, et pour lesquels les critiques hésitent entre diverses dénominations: «naïfs", «peintres du dimanche», «maîtres populaires de la réalité», "peintres de l'instinct», peintres «à l'état sauvage». Autant d'expressions auxquelles Uhde oppose, successivement, «peintres du Cœur sacré », «maîtres primitifs» et, enfin, «primitifs modernes", terme que retiendra l'historien de l'art Robert Goldwater. Le texte d'une dizaine de pages, très denses, qui reprend le récit de la rencontre avec Séraphine dessine les limites de son propre rôle: fournir les grandes toiles que réclame l'artiste et non les couleurs, qu'elle fait ellemême en gardant le secret de leur composition. Autodidacte que Unde n'a jamais vue peindre, sa technique a la perfection d'une vieille tradition de métier. Le thème du mysticisme médiéval est désormais libéré de la plaisanterie initiale - «peints par une nonne» - ou d'une assimilation trop directe à la piété religieuse. Mais, surtout, il est enrichi par une mise en correspondance entre un lieu, une histoire et une ferveur créatrice: le ciel de l'île-de-France, berceau des rois de France, la cathédrale de Senlis, alliance de l'esprit germanique et de l'esprit gallo-romain: «J'ignorais qu'ici le cœur sanctifié d'une servante se sentait la vocation de ressusciter le sublime du Moyen Âge et de créer des œuvres puissantes imprégnées d'esprit gothique. » (Uhde 2008: 70)

«Esprit», et non pas «style gothique»: la formule se fait l'écho des thèses que Uhde a longuement développées dans son Picasso et la tradition française et qui lui ont valu, dans le Paris de l'entre-deux-guerres, une certaine notoriété auprès des critiques et des historiens de l'art. La revue de Waldemar-George en a fait le point de départ d'une enquête sur «les origines de l'art gothique " dont les résultats ont paru dans ses deux premiers numéros, en 1929 et $1930^{21}$. La question gothique cristallisait alors des débats induits en histoire de l'art par l'affirmation des nationalismes et qui conduisaient, depuis le début $d u x^{e}$ siècle, à identifier les composantes françaises ou germaniques dans la qualification d'un style ${ }^{22}$. Dans son commentaire aux réponses reçues par la revue, Uhde rappelait le projet
19. Uhde 1929 ; Delteil 1930 ; Bing 1930; Waldemar-George $1931: 26$

20. Uhde 1931. Quelques œuvres de Séraphine côtoient des tableaux d'André Bauchant, Camille Bombois, Jean Ève, René Rimbert,

Rousseau, Maurice Utrillo et Louis Vivin. Elle est aussi présente dans l'exposition "Les maîtres populaires de la réalité ", à Paris et Zurich (1937), puis à Londres et New York (1938). Hans Körner et Manja Wilkens ont établi la liste complète des nombreuses expositions des années 1930-1950 (2009: 255-257).

21. La rédaction de Formes invite les lecteurs à répondre aux deux questions suivantes:

" $1^{\circ}$ Le gothique est-il une acception de style plastique et architectural, limité à une époque (un concept historique) ou la manifestation d'un état d'esprit? (un concept psychologique) $2^{\circ}$ Dans quelle mesure les Gallo-Romains et les Germains ont-ils contribué à la naissance et au développement du gothique? ", "Les origines de l'art gothique. Enquête", Formes, $\mathrm{n}^{\circ}$ 1, 1929: 12. Elle publie les réponses d'Émile Mâle, Josef Strzygowski, Louis Bréhier, Hans Karlinger, Konrad Escher, Élie Faure ainsi qu'une lettre ouverte de Uhde. Roland Recht a reconstruit les conditions historiographiques et idéologiques des thèses de Unde développées dans des textes inédits sur le "développement de l'homme européen dans l'esprit grec » ainsi que les deux perspectives que dessinent les réponses: recourir à des concepts nationalistes ou bien analyser le style en clé psychologique (Recht 2002-2003).

22. Sur les sympathies mussoliniennes de Waldemar-George qui, pourtant, se déclarait pacifiste et socialiste avant d'assigner au critique d'art une fonction de guide spirituel, voir Chevrefils Desbiolles (2008: 101-117); sur la reconnaissance de Strzygowski par les revues d'avant-garde parisiennes des années 1930 et la dimension raciale sousjacente à sa conception dualiste d'une histoire universelle des formes, voir Labrusse 2009. 


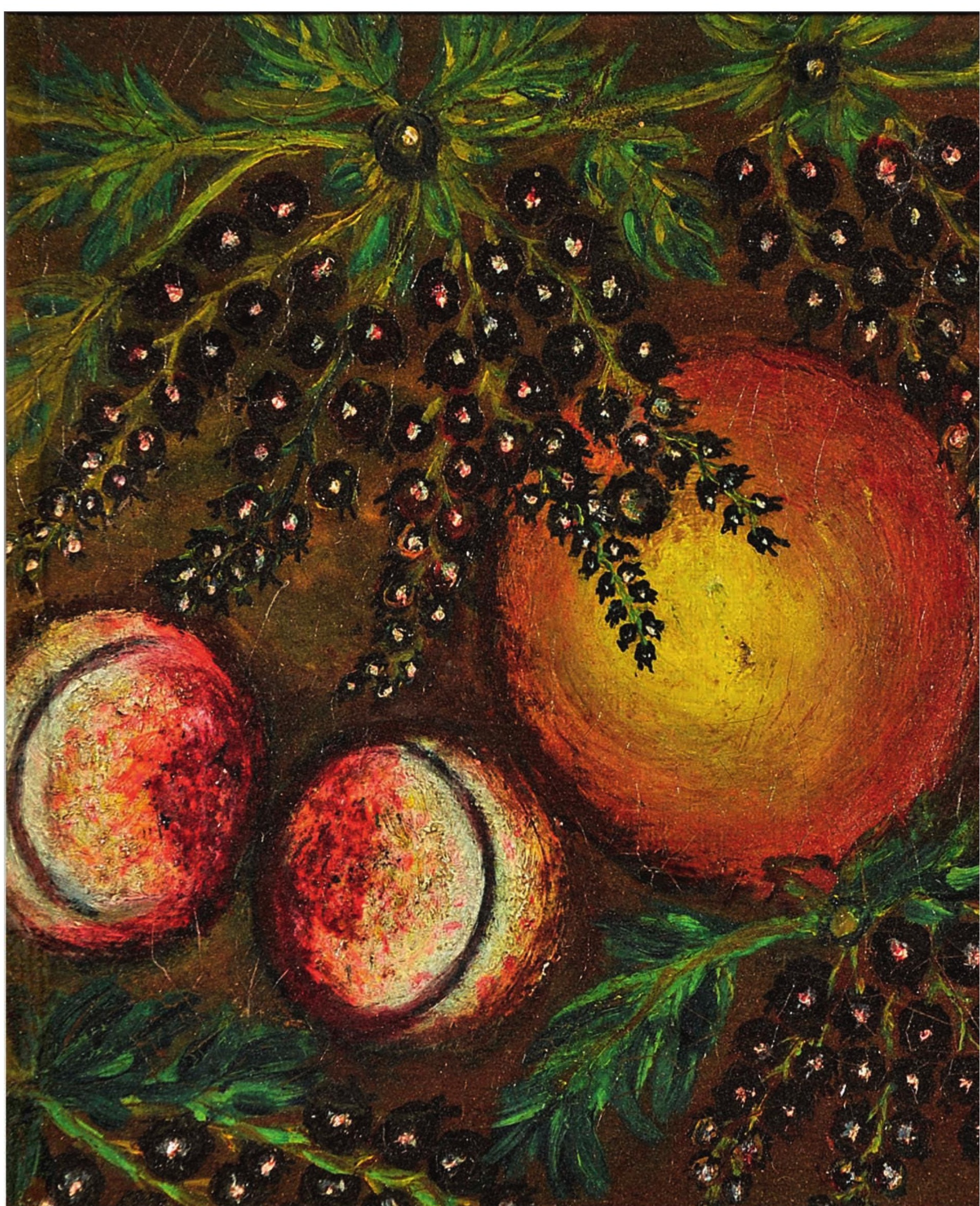




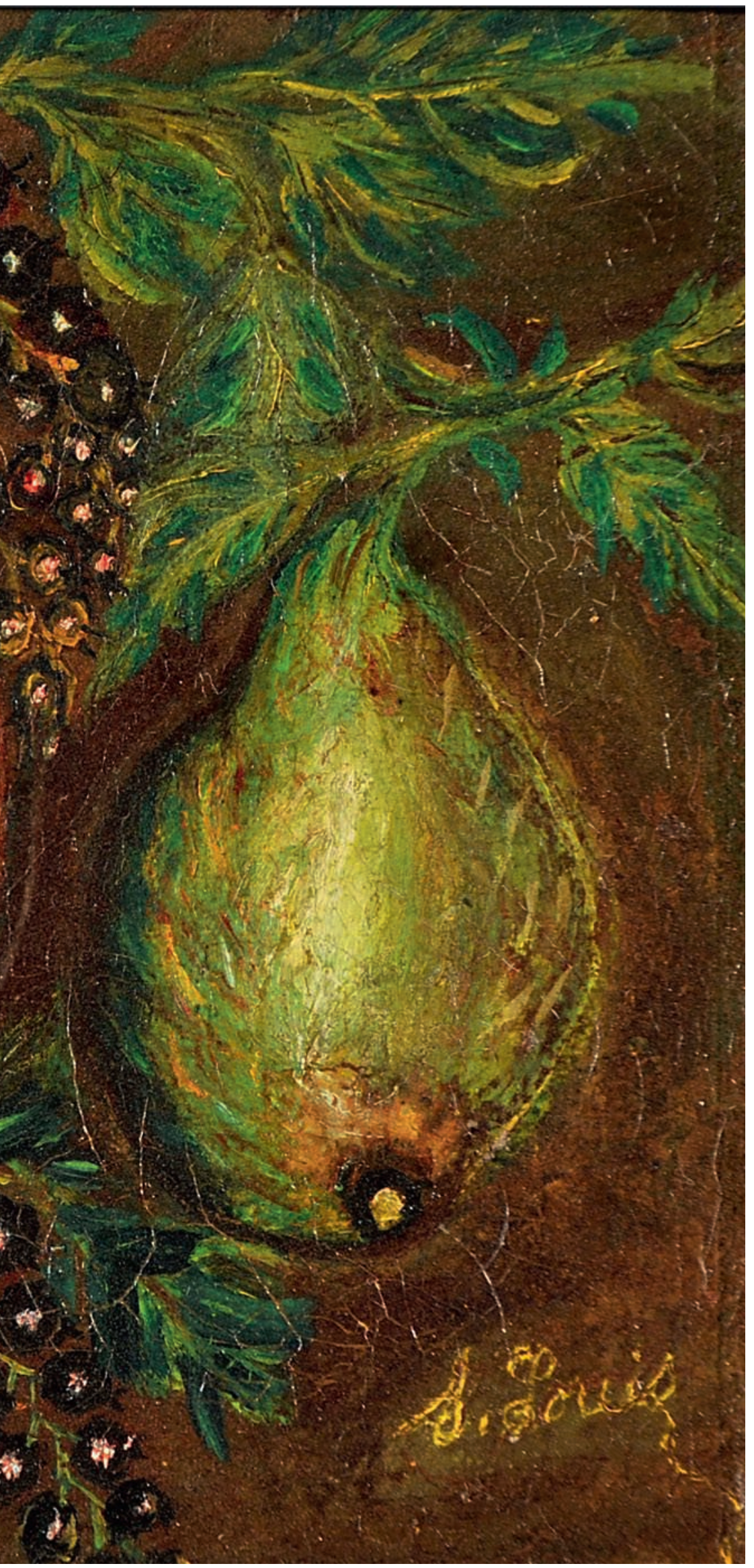

Senlis, Les Cassis, vers 1915

(C) Musées de Senlis.

Photo (C) Christian Schryve, Compiègne. 
poursuivi dans son livre: caractériser non tant un style historiquement daté qu' "un vouloir mystique qui se détourne du monde visible et s'oriente vers un monde invisible » - comme l'une des deux voies de la création artistique mise au service d'un nouvel humanisme.

Ennoblie en Séraphine de Senlis, la bergère d'Arsis devient la sœur cadette de la bergère de Domrémy, son prénom fait signe vers ces séraphins que l'on dit encore les embrasés, tandis que ses fleurs tourmentées et ses grands arbres de paradis apparenteraient cette «botanique spéciale à celle d'un Vincent Van Gogh. Bref, il faut être soi-même «en état de grâce pour comprendre cette œuvre, puis l'estimer et l'accepter» (ibid.: 79). L'effondrement dans la folie est évoqué avec une réserve pudique: la servante de l'esprit «vertical» a été, à son tour, emportée par cette fin du monde qu'elle s'était mise à prédire, de porte en porte, dans les rues de la vieille ville médiévale, dès lors que ses rêves d'opulence et la crise économique obligeaient Uhde à renoncer à son rôle de mécène.

\section{Le légendaire marial}

S'il identifie bien dans l'«inspiration» de Séraphine une ferveur religieuse en consonance avec la fonction spirituelle qu'il accorde à l'art comme substitut de la religion, Uhde ne s'est jamais fait le témoin d'injonctions à peindre ou dessiner émanant de la Vierge et/ou d'un ange gardien. S'il reconnaît une sorte de "sainteté» à l'œuvre dans ces transpositions picturales des rosaces et des tapisseries médiévales, il ne regarde pas Séraphine à travers ce catholicisme marial du xIx $\mathrm{e}^{\mathrm{e}}$ siècle que lui attribuent, trente ans plus tard, ses biographes et commentateurs. Mais le parallélisme suggéré par le critique d'art entre les deux bergères s'est rapidement transformé en un épisode biographique saillant. Le modèle de vie à l'envers qui sert localement à penser au féminin une marginalité sociale et psychique - c'est une "originale», un «peu simple», disait-on - a été mis au service d'un autre travail de singularisation: caractériser l'artiste inspirée. Quant aux essayistes en quête de «faits réels", ils ont traité ces motifs narratifs comme autant d'expansions des quelques métaphores employées par Uhde qui entendait, pour sa part, réhabiliter celle en laquelle les Senlisiens ne voyaient plus, justement, qu'une vieille femme démente.

Le premier enquêteur est le médecin psychiatre Henri Gallot que son grand-père Demoinet, adjoint au maire de Senlis, incitait, jeune homme, à s'intéresser à cette femme de ménage «un peu drôle» dont les petits tableaux n'étaient pas «si mal »... Elle aimait, lui disait-elle, les arbres, les fleurs, les oiseaux, et ramassait les plumes de faisan et de pintade en période de chasse. Peu à peu, elle avait consenti à parler peinture mais elle refusait obstinément de montrer ses œuvres. Ce n'est qu'un an après l'exposition locale de 1927 à l'origine des retrouvailles avec Uhde que Séraphine ouvrit au jeune médecin, un dimanche matin, la porte de son logement dans cette rue du Puits-Tiphaine habitée par les laveuses et les repasseuses. On doit à ce dernier une description précise de la plus grande pièce qui lui servait d'atelier: «La salle était assez vaste sans autres meubles qu'une table de bois blanc, deux ou trois chaises, et une petite armoire basse sur laquelle était une statue de la Vierge. Devant cette statue une veilleuse à huile était allumée. Çà et là, quelques roses de papier étaient épinglées. 
De très grandes toiles étaient appuyées contre les murs mais retournées "à cause de la poussière". Le plancher était impeccablement propre, non ciré, simplement lavé. [...] Nulle part je ne vis de couleurs ni de palette. Dans un vase près de la statue, il y avait quelques pinceaux. Comme je m'étonnais, elle me répondit simplement: "Tout est rangé, j'ai ma manière ${ }^{\mathbf{2 3}}$." ” II ne la reverra plus jamais: à son retour à Senlis, pour les fêtes de Pâques 1932, Séraphine était internée depuis deux mois. L'idée d'une enquête auprès des voisins et dans les archives lui vint «bien des années plus tard». Le long article qu'il publie dans L'Information artistique, en mai 1957, et qu'il reprend partiellement en 1968 compose le portrait, physique et psychique, d'une «originale», "vêtue de manière anachronique», dont les chroniqueurs suivants vont amplifier les traits selon la logique qualifiant une féminité désordonnée. Sa jupe longue, sa pèlerine grise, ses cheveux frisés coupés court, son canotier et ses souliers d'homme vont se transformer en autant d'extravagances vestimentaires; et alors que le médecin souligne simplement ses manières de parler - une voix aiguë, avec un curieux accent picard -, on insistera sur ses sautes d'humeur et ses relations difficiles, à l'église, avec le sacristain et le curé qu'elle interpelle en plein sermon ${ }^{\mathbf{2 4}}$. Gallot est aussi le premier à introduire un motif - l'invitation surnaturelle à dessiner dont l'expansion ne cessera de croître. Sans doute ne l'a-t-il pas inventé: les divers certificats d'internement, au début de 1932, mentionnent des "visions de l'au-delà » et les voix de Dieu, de la Vierge, de sa sœur décédée. Début novembre 1933, un interne de l'asile de Clermont-de-l'Oise note les propos que tient alors Séraphine: «En 1905 en étant chez M. Chambard, son ange gardien lui aurait dit à l'église de se mettre aux dessins. Elle commençait alors à dessiner des fleurs, des fruits sur papier spécial, sur des vases, cruchons, bouteilles et assiettes ${ }^{25}$. "Gallot en fait, tout d'abord, le trait distinctif d'une créatrice indemne de toute éducation artistique, autant dire d'une créatrice inspirée qui fête, chez elle, sainte Thérèse de l'Enfant-Jésus en décorant sa chambre de guirlandes de roses en papier. L'urgence qui s'empare de Séraphine lorsqu'elle quitte brusquement son travail pour regagner sa chambre répond aux exigences de ses visions. Mais, en psychiatre, Gallot tente aussi de mettre en parallèle états psychiques et manières de peindre. Si l'art, techniquement très sûr, de Séraphine lui permet «d'extérioriser symboliquement» des perceptions délirantes pour produire, entre 1928 et 1931, les chefs-d'œuvre qui enthousiasment Uhde, on ne saurait pour autant voir dans la folie un état favorable à l'invention artistique, et encore moins assimiler «l'étrange splendeur» des derniers tableaux aux médiocres productions des malades qui se mettent à peindre.

Dix ans plus tard, cette interrogation clinique sur les rapports entre création et folie fait place, avec Jean-Pierre Foucher, à un légendaire hagiographique $^{26}$. Reprenant la vie de Séraphine, il introduit le motif que l'on attribue à Giotto depuis la Renaissance: enfant, tandis qu'elle garde les bêtes des voisins, elle dessine en secret des scènes rustiques que les écoliers viennent admirer. Entrée à 18 ans comme domestique chez les sœurs de Saint-Joseph de Cluny, elle aurait aspiré à devenir religieuse jusqu'à ce qu'un autre appel l'incite, vingt ans plus tard, à quitter le couvent ${ }^{27}$. Un appel auquel l'auteur donne la forme concrète d'un dialogue avec l'ange dans la cathédrale - «Séraphine, écoute-moi: il faut te mettre au dessin» - puis d'un théâtre d'apparitions mariales joué par les ouvrières de l'atelier de cou-
23. Gallot 1968 : 66 À comparer avec le témoignage d'Anne-Marie Unde: "C'est en 1929 que pour la première fois j'accompagnai mon frère chez Séraphine à Senlis.

Je la trouvai dans une modeste chambrette. Le mobilier se composait d'un lit, de deux chaises, d'un petit fourneau et d'un chevalet. Sur la cheminée brûlait une petite lampe devant une statuette de la sainte Vierge. Les murs étaient couverts de peintures commencées, d'une puissance saisissante.

Et au milieu de ces toiles extraordinaires, se tenait une petite vieille femme, un peu fanée, un peu chétive, dont le visage et surtout les yeux exprimaient une vie intérieure ardente", in La Réalité intérieure. Séraphine et quelques peintres autodidactes. Donations de W. et A.M. Unde aux musées français, Senlis, musée d'Art et d'Archéologie, 1989: 2.

\section{Les souvenirs} d'enfance d'Henri Leblanc (1972), illustrateur-graveur qui, jeune homme, a dessiné à plusieurs reprises celle que l'on surnomme «la peintresse", attestent la fascination exercée sur les jeunes garçons: "Séraphine pour les gamins de mon âge, c'était une petite vieille bizarre, aux cheveux vaguement roux, frisés et coupés à l'artiste au niveau du cou [...]. Elle était peu bavarde et le timbre fort aigu de sa voix était aussi surprenant que les rares regards qu'elle posait sur vous, de ses yeux à l'iris changeant bleu-vert pailleté de jaune. "Sur les formes masculines et féminines de vies désordonnées, je me permets de renvoyer à Charuty 1997.
25. Observation médicale citée par le Dr Marie-Amélie Ortas-Peretti (1965: 11).

26. Spécialiste de littérature latine médiévale, Jean-Pierre Foucher préface également les grands textes de la matière de Bretagne pour Gallimard et s'intéresse aux contes ossianiques de Chateaubriand.

27. On sait désormais qu'il s'agit du couvent de la Charité de la Providence à Clermontde-l'Oise. 


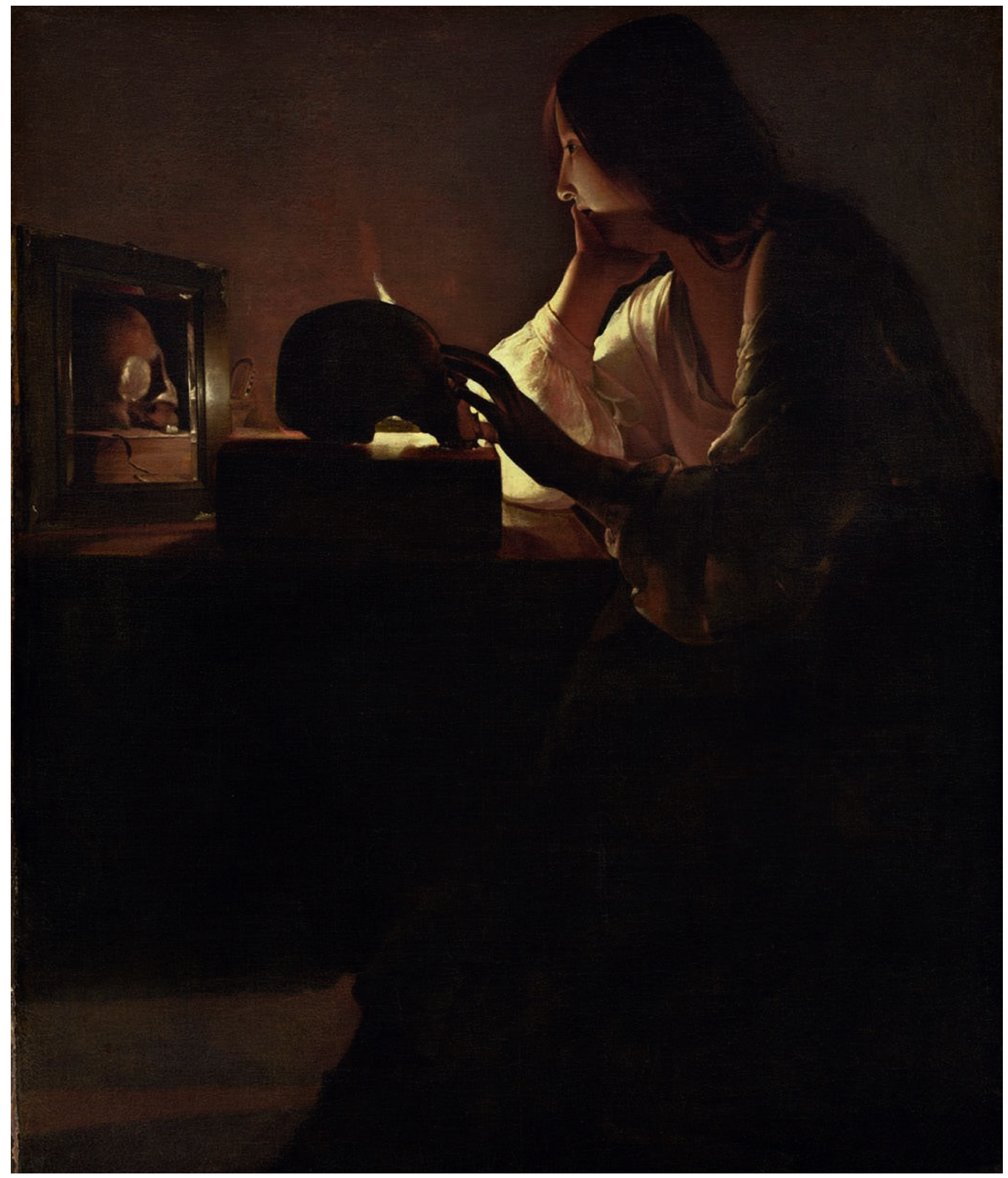


ture local, selon une sorte de légendaire oral que l'écrit contribue à fixer: «Elle ne se fâchait pas quand les ouvrières de $\mathrm{M}^{\mathrm{me}}$ Dufflos-Haute couture complotaient de petites farces à son intention: I'une d'elles disparaissait de l'atelier (c'était Odette ou Madeleine), s'habillait en sainte Vierge avec une ceinture bleue et un voile de tulle et "apparaissait" à Séraphine pour lui dire: "Séraphine, ton fiancé revient cette semaine" ou bien: "Je suis la sainte Vierge et je te dis, Séraphine, que tu seras un grand peintre." Elle venait, elle regardait. Elle hochait la tête. Elle disait: "Petites sottes, vous n'y connaissez rien. Vous ne savez pas ce que c'est! Ah, si vous pouviez voir comme c'est beau, quand Elle vient!" "(Foucher 1968: 17, 28) Le portrait physique et moral de l'artiste accentue le motif de la pauvreté: «Une petite femme sans âge, encombrée de jupes, de caracos, portant un éternel châle court alors appelé "palatine", coiffée d'un canotier qu'elle repeignait au ripolin noir, à la main un cabas dans lequel elle enfouissait les vagues nourritures qu'elle achetait ou que, plus souvent, ses pratiques lui donnaient à emporter. » (Ibid.: 27-28) Une apparence misérable qui contraste avec l'acharnement à peindre, jour et nuit, en chantant les cantiques appris durant les vingt ans de vie recluse: «Elle peint à genoux, sa toile posée à plat sur le plancher, s'éclairant de la seule lampe pigeon. Et, le jour, qu'on ne s'avise pas de la déranger. Qu'on respecte l'injonction des trois écriteaux qu'elle a installés sur le chemin d'éventuels visiteurs: "Défense de monter sous peine de poursuites. Je travaille." Elle s'enferme alors derrière tout un attirail de loquets, de verrous et de chaînes. » (Ibid. : 37)

Les deux biographies d'Alain Vircondelet accentuent le catholicisme marial de cette composition narrative en identifiant, de manière caricaturale, Séraphine à une nouvelle Bernadette de Lourdes. L'ange gardien s'est transformé en saint Michel, doublé de la Vierge, dans une «Annonciation» qui assimile l'entrée en peinture à la véritable entrée au couvent. Ses principales interlocutrices terrestres sont les religieuses auxquelles, devenue insupportablement bavarde, Séraphine adresse d'improbables monologues sur l'équivalence entre peindre et prier: «Quand je prie maintenant, c'est quand les fleurs naissent de mon pinceau. Je me dis, tiens, voilà une fleur de plus dans le paradis du Bon Dieu. Et j'en fais plein, vous savez, j'en fais. » (Vircondelet 1986: 68) Peindre à genoux devient le signe d'humilité de la servante de Dieu, et cette mièvrerie saint-sulpicienne alterne avec des scènes d'exaltation mystique lues, il fallait s'y attendre, en clé d'hystérisation. Aux injonctions du ciel, le biographe ajoute des messages médiumniques qui soumettent l'acte de peindre à une dictée surnaturelle. Lue comme la «passion » de Séraphine, l'entrée dans la folie occupe la moitié du second récit, qui expose longuement les thèmes délirants présents dans l'écriture épistolaire qui a remplacé la peinture à l'asile.

Bien qu'écrit dans un registre beaucoup moins caricatural, la «vie rêvée» par Françoise Cloarec s'ouvre, d'emblée, sur le motif de l'ordre surnaturel: "Séraphine tu dois te mettre à dessiner. ( (Cloarec 2008: 11) Puis l'auteur déroule le récit d'une enfance pieuse et solitaire avant de reproduire l'injonction mariale à se consacrer à un travail de figuration qu'elle entend éclairer en psychanalyste: ne pouvant s'autoriser elle-même à peindre, Séraphine convoque une instance extérieure, ange ou Vierge selon les versions. Aussi bien, les journalistes qui rendent compte du film reprennent-ils tous

\section{ci-contre}

fig. 6

Georges de la Tour, La Madeleine au miroir, vers 1635-1640. National Gallery of Art, Washington. Ailsa Mellon Bruce Fund 1974.52.1. 
28. Dossier

d'accompagnement

pédagogique, www.

seraphine-lefilm.com.

29. "Cinéma: Martin Provost nous parle de Séraphine ", www.dailymotion.com,

14 avril 2009

30. Philippe Vandendriessche, entretien filmé, www.cinergie. be/webzine/philippe_ vandriessche (consulté le 17 mai 2012).

31. Cette découverte du tableau anonyme acheté chez un commerçant du boulevard de Rochechouart, un jour de 1905, et l'identification, quelques jours après, au Lapin Agile, par son auteur, Picasso, est présentée par le collectionneur comme le début d'une profonde amitié (Uhde 2002: 137). le motif de l'apparition ou du message surnaturels pour mettre en énigme la pulsion créatrice de Séraphine. Ils y sont, de plus, expressément invités par le dossier pédagogique qui incite les enseignants à faire du film une leçon d'histoire des relations entre vécu religieux et création. Soulignée par la mise en page, la «révélation» de Séraphine acquiert la consistance d'une expérience mystique qui serait commune à d'autres créateurs contemporains - tel Max Jacob converti par l'apparition, en 1909, du visage du Christ sur le mur de sa chambre - dont l'œuvre porterait, dès lors, la marque ${ }^{\mathbf{2 8}}$.

Mais qu'en est-il dans la narration filmée?

\section{La conversion artiste}

Ponctué par quatre indications de lieu et de temps - Senlis, 1914; Chantilly, 1927; asile de Clermont, 1935; asile de Clermont, 1942 -, le film transpose, certes, des scènes de ces diverses narrations. Mais c'est l'entière trajectoire de l'entrée de Séraphine dans la peinture que le cinéaste recompose à travers l'accent porté sur la rencontre avec le collectionneur. Soulignons, d'abord, le souci de "vérité», de "sincérité », d'«authenticité » qui revient, comme un leitmotiv, chez le réalisateur, les acteurs, l'ingénieur du son. Un souci qui a conduit le cinéaste à engager une «actrice métaphysique" avant d'écrire le scénario et à mettre à profit les aléas de la production pour vivre avec elle une sorte de pèlerinage ludique sur les traces de l'artiste. «On a passé énormément de temps ensemble. On s'est vus pendant un an avant de tourner. On est allés voir les tableaux, on s'est promenés dans Senlis, je l'ai emmenée voir la maison de Séraphine, on a monté les escaliers, on a frappé à sa porte et puis... que dalle, bien sûr! [...] J'ai montré les arbres, on s'est promenés dans les forêts de bouleaux. Ça a été un travail d'approche pour qu'on soit, au tournage, excessivement proches l'un de l'autre ${ }^{29}$."

Souvent, les tableaux que donnent à voir les films de peintres sont de simples reproductions photographiques: ici, d'abord transposés en photographie par impression numérique sur toile, au format réel, ils ont été repeints à la manière de l'artiste par un peintre décorateur. L'ingénieur du son souligne à son tour: «Les acteurs savaient jouer quand ils jouaient, savaient chanter quand ils chantaient, savaient peindre quand ils peignaient ${ }^{30}$.» À quoi fait écho l'actrice: «J'ai pris des cours de peinture pour obtenir une crédibilité dans le geste. J'aimais dire: "Je rentre dans les pommes de Séraphine." "Ajoutons que Yolande Moreau a appris à chanter des cantiques en latin avec un prêtre voisin, le curé de Vernon. À ce souci de réalisme répond le souci esthétique et didactique de reconstruire l'univers pictural de la collection de "primitifs modernes» rassemblée par Unde: Rousseau, Bombois, Vivin sont présents dans la maison de Chantilly tandis qu'un dessin de Picasso rappelle le premier achat d'un tableau signé seulement de l'initiale du peintre ${ }^{31}$.

Mais ce parti pris réaliste est enchâssé dans une atmosphère onirique produite par des moyens modestes, assez conventionnels - les coupures du montage, les décrochages, les ellipses qui brisent la logique narrative ou par une manière, plus surprenante, de recréer l'équivalent d'une peinture «primitive». Ainsi de la couleur comme en aplat ou des plans pensés 
comme des tableaux: le corps de Séraphine se confond avec les branches tordues d'un arbre dont l'ampleur et la majesté sont accentuées par le cadrage qui l'isole et le découpe sur le vert cru du pré et le gris bleuté du ciel. La caméra qui s'arrête, un instant, sur les jambes épaisses de Séraphine comme Bombois peint en gros plan les lourdes cuisses de ses personnages féminins restitue au spectateur le regard que Unde pouvait porter d'un primitif à l'autre.

Le motif de l'injonction de l'ange ou de la Vierge est certes présent, mais le plus souvent de manière indirecte, comme un discours rapporté par chacun des interlocuteurs de Séraphine. «Elle travaillait au couvent à côté quand son ange gardien lui est apparu et lui a ordonné de se mettre à la peinture..." ", déclare $\mathrm{M}^{\mathrm{me}}$ Duflos à ses invités. Puis, un peu plus tard, s'adressant à Unde: «À propos, elle vous a parlé de son ange gardien?" "Tu es sûre, Séraphine, que c'est ton ange?", interrogent les religieuses effarées par des végétations aussi échevelées. En somme, ce motif n'est donné à entendre qu'à titre de catégorie disponible dans le monde social de Senlis: s'il peut susciter des sourires apitoyés, des ricanements ou des interrogations inquiètes, l'énoncé est de l'ordre du dicible et du "croyable ". Face à ce registre de l'herméneutique locale, un autre appel est donné à voir au spectateur. Prenant la place de l'ange annonciateur, le collectionneur s'efforce d'arracher Séraphine à son travail ménager dans une scène qui inverse la composition visuelle de la scène religieuse: accroupi sur ses talons, Uhde lève la tête vers Séraphine qu'il a fait asseoir de force sur une chaise pour la persuader qu'elle a, en elle, les ressources de l'art. «Vous êtes douée, mais il va falloir travailler beaucoup", assure-t-il. La seconde visite de l'«ange" Uhde est celle que provoque, après guerre, l'exposition à l'hôtel de ville de Senlis: debout, de profil, sur le seuil de la misérable chambre-atelier, il profère le jugement décisif: «J'ai vu votre travail, vous avez fait de grands progrès. »

La naissance de l'artiste et la transformation de sa technique sont données à voir comme une conversion en trois actes. Dans un premier temps, le cinéaste libère Séraphine de toute interprétation psychologique pour lui donner des mains, un regard et un corps. Le mouvement de la caméra restitue la tension qui traverse ce corps, déplacé entre le trop bas - tout ce qui se passe au ras du sol - et le trop haut - tout ce qui se voit dans le ciel. Elle est agenouillée à terre, tantôt le regard absorbé par le sol à laver ou à cirer, ou par la surface à peindre et à colorer, tantôt la tête levée, le regard tourné vers la maîtresse de maison, vers la Vierge, vers le ciel. La caméra donne à voir des mains qui s'affairent, avec la même énergie, aux activités ménagères, aux préparatifs de la peinture et à la prière. Mais il ne s'agit pas simplement de soustraire le geste de création au champ de l'illusion en le replaçant dans la continuité des gestes quotidiens.

Se laisser glisser sur les grandes pentes d'herbe verte, arracher par poignées boutons d'or et coquelicots qui laissent des taches de couleur sur la peau, escalader ou enlacer les arbres, jouer avec les effets de la lumière solaire filtrée par les feuilles, analogues aux effets de lumière à travers les vitraux de la cathédrale: ce sont autant d'explorations sensorielles que l'on retrouve dans les récits d'enfance paysanne où tout élément peut être dé- 


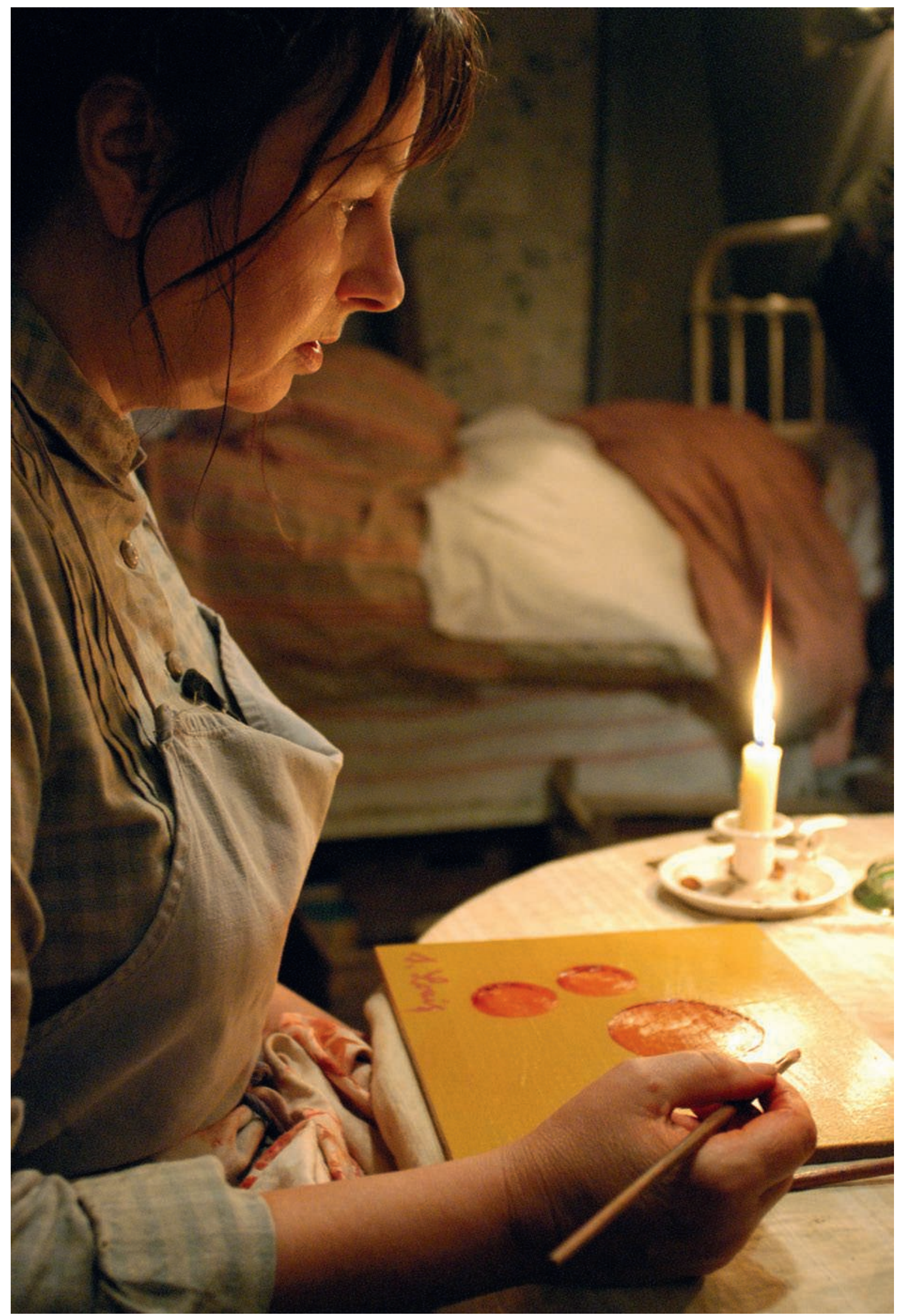


tourné de son usage premier pour recomposer un univers signifiant. Les traits de conduite qui, dans le discours local, assimilent Séraphine à une originale, une innocente, bref un être déplacé, deviennent l'actualisation de cette perturbation méthodique. Créer c'est alors entrer, comme les enfants, dans un rapport prédateur à la nature, aux hommes et à l'Église, pour collecter les ingrédients - boues colorées, écorces, racines, plantes, fleurs, huile et sang - qui, détournés de leur valeur d'usage, vont pouvoir entrer en une composition, quasi culinaire, de l'image peinte: c'est la première séquence, où elle prépare les couleurs avec des gestes de ménagère, broyant dans un mortier tous les ingrédients ramassés dans ses pérégrinations. Une ménagère un peu sorcière qui, sous le regard de l'esthète allemand, semble se transformer en fée Mélusine, comme le suggère le bain solitaire dans la rivière filmé à travers le regard étonné de Uhde.

Par opposition à la quête prédatrice, l'acte de peinture exige le retrait du monde extérieur pour instaurer, dans le silence et la solitude nocturne, un face à face avec la Vierge où s'affirme non l'équivalence générique entre peindre et prier mais la mise en mouvement de la main par la voix chantée. Peindre, c'est louer la petite Vierge de Lourdes, illuminée de bougies sur l'autel domestique, le rythme du chant liturgique accompagnant le rythme de la main qui étire directement la pâte colorée, sans la médiation d'un outil. Puis c'est le regard, dissocié de la main, que la caméra offre à la contemplation du spectateur pour instaurer, dans ce regarder voir, l'équivalence du voir et du toucher et, ce faisant, éprouver la consistance de cette «pâte » qui a retenu l'attention de Uhde. Cette convocation de la peinture dans l'image cinématographique se réalise ici non dans la réalisation fictive d'une œuvre de l'artiste, mais en faisant surgir chez le spectateur la mémoire des tableaux d'un grand primitif, redécouvert au même moment, Georges de La Tour. Un choix judicieux puisque c'est un historien de l'art allemand - Hermann Voss - qui a l'initiative, en 1915, du travail d'attribution qui aboutira à la grande exposition Les Peintres de la réalité en France au XVII siècle, au musée de l'Orangerie, de novembre 1934 à février 1935. Comme le peintre lorrain faisant surgir des êtres «divisés entre la nuit où ils s'élèvent et la lueur qui les éclaire en partie ", Provost substitue le reflet d'une bougie à tous les récits d'appel surnaturel ${ }^{32}$.

La deuxième séquence a lieu durant la guerre, après la première rencontre et la fuite de Uhde. Peindre commence à nouveau par un acte de prédation, dévaliser la droguerie Duval de la place du Marché pendant l'offensive allemande; par la solitude dans Senlis désertée au milieu des éclats d'engins explosifs et des gerbes de lumière. Mais Séraphine est absorbée dans une autre guerre: celle qu'elle livre, à genoux, échevelée, le tablier maculé de peinture, à la surface de grands formats de toile d'où elle fait surgir des gerbes colorées, dans une sorte de frénésie, les yeux fixes et brillants, les gestes rapides et sûrs, soutenue par son vin d'«énergie " pour défaire dans l'ivresse la domestication des exercices spirituels. Nous ne sommes plus dans le secret de la nuit, dans l'atmosphère de dévotion à la Vierge, la chaude lumière des bougies s'est éteinte sous la grisaille du jour. Au regard contemplatif succède l'inquiétant pouvoir visionnaire auquel accède l'artiste dans une sorte d'auto-hypnose.
32. Reflet que Pascal Quignard qualifie de "singulier reflet de grandeur" dans son beau commentaire de la manière de Georges de La Tour (2005 [1991]: 20 et 32).

\section{ci-contre}

fig. 7

Yolande Moreau interprétant

Séraphine Louis dans

le film Séraphine de

Martin Provost, 2008.

Production TS Productions.

Distribution Diaphana.

Photo (c) Michaël Crotto. 
Les deux seules photographies que nous ayons de Séraphine en artiste peintre la représentent debout en blouse noire, la palette à la main, devant une toile dressée à la verticale. La première a été prise par un artisan de Senlis, Albert Benoît, tapissier décorateur qui revendique l'antériorité dans la reconnaissance de son talent. L'autre, plus connue, prise par Anne-Marie Uhde dans la seconde moitié des années 1920, nous montre un visage tourné vers le haut, les yeux fermés: «Il faut que je lève le front. Mon inspiration vient d'en haut », a expliqué l'artiste. La troisième séquence de la fiction cinématographique transpose à sa façon ce travail d'identification réalisé, près d'un siècle avant, par la photographie. Elle succède aux retrouvailles avec Unde, au regard d'expert qu'il a posé sur les toiles, à la reconnaissance d'un métier et d'un talent que devrait consacrer une exposition qui serait entièrement consacrée à l'artiste. Cette exposition qui n'a pas eu lieu de son vivant, le film nous l'offre, réalisée par Séraphine elle-même. L'image de la main qui signe la toile encore vierge introduit à la métamorphose finale d'une vie placée «sous l'emprise du tableau », comme le voulait Uhde. Ce que donne à voir le «ballet des tableaux» présenté de manière théâtrale devant une rangée de chandelles - comme une projection de lanterne magique, indique le synopsis - aux voisines, à la religieuse et à Anne-Marie Unde (Provost s. d. : 91). Entrée dans le tableau, l'artiste joue à cache-cache avec nous, comme cet œil qui nous regarde, enfoui dans les fleurs et les feuillages de ses arbres de paradis: non le peintre en personne, mais le tableau en personne. Et la puissance créatrice qui l'anime conserve une part d'obscurité: «Moi aussi j'ai peur parfois de ce que j'ai fait. »

Le motif du fiancé imaginaire qui, dans les récits biographiques, est toujours rapporté comme un signe d'extravagance - dans la logique de ces vies à l'envers qui font du mariage le critère d'une féminité ordonnée se trouve, ici, mis au service d'une interrogation sur le ressort psychique de la passion créatrice: «Quand on fait de la peinture, mademoiselle, on aime autrement. " Séraphine a désormais les postures, la gestualité et les instruments du peintre, selon les représentations les plus conventionnelles: on la voit de dos travailler debout, une palette à la main, revêtue d'une longue blouse de peintre, devant une toile de grand format posée sur un chevalet dont la hauteur dépasse sa taille. La Vierge est toujours là mais l'artiste lui tourne aussi le dos, absorbée dans le frottement du pinceau sur la toile dont le bruit sec a remplacé les cantiques de louange. Un autre chant s'élève qui prend, cette fois, les accents sauvages d'une danse de possession: peindre est un corps à corps avec le tableau qu'elle prend dans ses bras, dépose à terre, interpelle avec des gestes d'incantation. Mais cette séquence se termine par une scène à la signification incertaine: ce qui aurait pu être l'enfantement d'une œuvre fait signe vers un effondrement car si, privée de regard, la peinture est impuissante à produire un monde, alors c'est le monde lui-même qui est appelé à s'effondrer.

\section{Apocalypse peinte, vécue, filmée}

Dans le silence de l'aube, une mariée en austère robe blanche, enveloppée d'un voile retenu sur la tête par une couronne de roses blanches, parcourt les rues endormies de Senlis. D'une main elle tient un lourd baluchon de drap aussi blanc que sa longue robe. De l'autre, elle frappe aux portes closes à l'aide d'un lourd chandelier d'argent et mur- 
mure, aux visages étonnés qu'elle a réveillés: "Mes tableaux sont bénis, mes tableaux sont bénis, c'est la fin du monde. " Tout en haut d'une ruelle gravie d'un pas lourd que rien ne semble pouvoir arrêter, deux hommes en noir l'attendent en silence; avec précaution ils la prennent par le bras, la font monter dans un fourgon noir qui démarre aussitôt. Reste dans la mémoire du spectateur le visage au regard fixe de la mariée, derrière les barreaux du fourgon.

Séraphine est internée à l'asile de Clermont-de-l'Oise en 1932. Trente ans plus tard, une jeune psychiatre, incitée par Jean Delay à interroger les rapports entre création et folie, retrouve dans les archives de l'hôpital les communiqués menaçants qu'elle a, des années durant, adressés à la "Très Haute Police de la Gendarmerie» de Clermont ou de Beauvais. Ils nous permettent de mesurer les ressources offertes à l'artiste par l'apocalyptique chrétienne pour donner sens à l'effondrement de ses rêves de reconnaissance. En communication directe avec Dieu comme avec Satan, Séraphine annonce les épreuves et les morts à venir, incite les uns et les autres à la repentance, et, surtout, proclame le rôle décisif qui lui a été dévolu depuis la création du monde: assurer la fonction salvatrice délaissée par la Vierge qui a succombé à Satan.

Nous sommes sous le règne de Sâtan, c'est êpouvantable. Les vertûs des cieux seront ébranlés. Les étoiles tomberont, Sâtan arrivera à tentés les élus puisqu'il est dit ceux qui seront êprouvés manqueront de pain, Sâtan changera les pierres en pain pour les enfants des ténèbres. Mais les enfants de Dieu qui s'appelle les enfants de lumières pour les autres il tombera à nouveaux de la mâne des cieux. II ma dit Sâtan je souillerais la Mère de D.S.C. Et j'y ferais ma demeure et je la détrôneraie effronté qu'il est et si vous le dite, je vous pûniraie.

[...] D. quand j'étais jeune, m'a interdit le mariage pour me saûver p.m.s. Et actuellement il m'en demande l'obligation pour me tenire saûver. Dieu me dit qu'il ajoutais encore avant la fin du monde 1000 ans et plus parce qu'il y a trop d'âmes à venire sur la terre et il faut leur en donner le temps, des millions d'âmes; d'enfants ils faut qu'ils viennent pour se sanctifié pour être sauvé; c'est-à-dire gagner le ciel, car alors au ciels il faut gagner sa place venir sur la terre pour y souffrir. (Ortas-Peretti 1965: 10)

Les divers motifs qu'entrelace cette fièvre eschatologique témoignent, bien sûr, d'une évidente familiarité avec les récits chrétiens. Cependant, la critique d'art allemande la plus récente a cru pouvoir identifier dans l'œuvre de Séraphine un «tournant iconographique vers l'apocalypse", bien avant la crise qui a provoqué l'internement et le délire que celui-ci n'a fait qu'amplifier (Körner et Wilkens 2009: 20). En particulier, le plus célèbre, le plus admiré et le plus inquiétant de ses tableaux, L'Arbre de paradis - nom que lui a attribué Unde -, associerait le motif de l'arbre de vie au Christ, présent de manière métonymique à travers l'œil que l'on distingue au milieu des feuilles au contour ovale, à l'aspect aérien de plumes. Tous les arbres de vie ou de paradis peints par Séraphine ainsi que les fleuves et les végétations luxuriantes des tableaux de grand format sont, dès 
lors, lus en clé apocalyptique: ce seraient autant de représentations de la Jérusalem céleste.

On ne discutera pas ici le bien-fondé d'une lecture rétrospective, fondée trop souvent sur des hypothèses invérifiables. En revanche, attachons-nous aux solutions visuelles que le cinéaste a, à son tour, adoptées pour signifier l'imaginaire apocalyptique attesté par le dossier médical. Sous la forme d'un arbre immense qui se détache au loin sur l'horizon, que Séraphine escalade pour s'installer entre ciel et terre, qu'elle entoure de ses bras pour mieux lui parler ou sous lequel elle va s'asseoir pour échapper à l'asile, l'arbre de vie ou de paradis ponctue le film de sa sombre présence, tour à tour accueillante et inquiétante. Des scènes hallucinatoires brièvement décrites dans le synopsis devaient, en outre, signaler l'entrée dans la folie. Séraphine entend des voix qui semblent provenir d'un placard, elle l'ouvre et voit un jeune homme mort dans un champ, la tête en sang, en costume de marié, qui tend la main. Ou encore, elle voit $\mathrm{M}^{\text {me }}$ Duflos couchée sur son lit, qui lui tend les bras pour qu'elle la réchauffe. Quant à la déambulation dans la ville endormie, le cinéaste l'imaginait ainsi: elle frappe à la porte d'une maison, tend un coquetier, des couteaux, des fourchettes en disant: «Prenez, prenez, car ceci est mon corps; prenez, prenez, car ceci est mon sang. Mes tableaux sont bénis, mes tableaux sont bénis, c'est la fin du monde. » (Prévost s.d.: 105)

S'écartant délibérément de tout diagnostic psychiatrique, le cinéaste choisit finalement de mettre l'accent sur la dimension culturelle de cette "expérience vécue de fin du monde», comme auraient dit les phénoménologues qui, entre 1920 et 1950, cherchaient à décrire des mondes vécus en deçà du partage entre normal et pathologique. Au tournage - ou au montage -, la sobriété s'est imposée pour restituer le caractère onirique d'une apparition: celle d'une Séraphine - Vierge de l'Apocalypse, au bras armé de ce candélabre qu'évoquent les textes bibliques, qui fait ouvrir les portes des maisons endormies et, comme dans les contes, laisse derrière elle des objets incongrus pour assurer les dormeurs qu'ils n'ont point rêvé. Et c'est par le silence qui enveloppe sa lourde silhouette blanche que nous, spectateurs, participons de la catastrophe du monde que tente de retarder cette Vierge soustraite au regard des hommes qui ne voient que catastrophe intérieure.

Quel est l'intérêt, pour nous, de ce regard de la fiction sur l'acte de peindre? Non pas tant d'accéder à une vérité que laisserait échapper la rigueur historiographique. Mais, plutôt, d'actualiser une modalité contemporaine de relancer l'imaginaire du «mystère de la création»: l'ange gardien et le collectionneur incarnent bien deux manières antagonistes de qualifier l'acte créateur - la main de Dieu ou le savoir-faire des hommes, à leurs risques et périls - et deux formes de transcendance - l'une chrétienne, l'autre à rattacher à cette religion de l'art qui, depuis deux siècles, se propose d'en prendre la relève. Mais la ruse du troisième ange de Séraphine, le cinéaste, est sans doute de faire coexister dans la même œuvre ces deux perspectives dont il condense les principaux éléments 


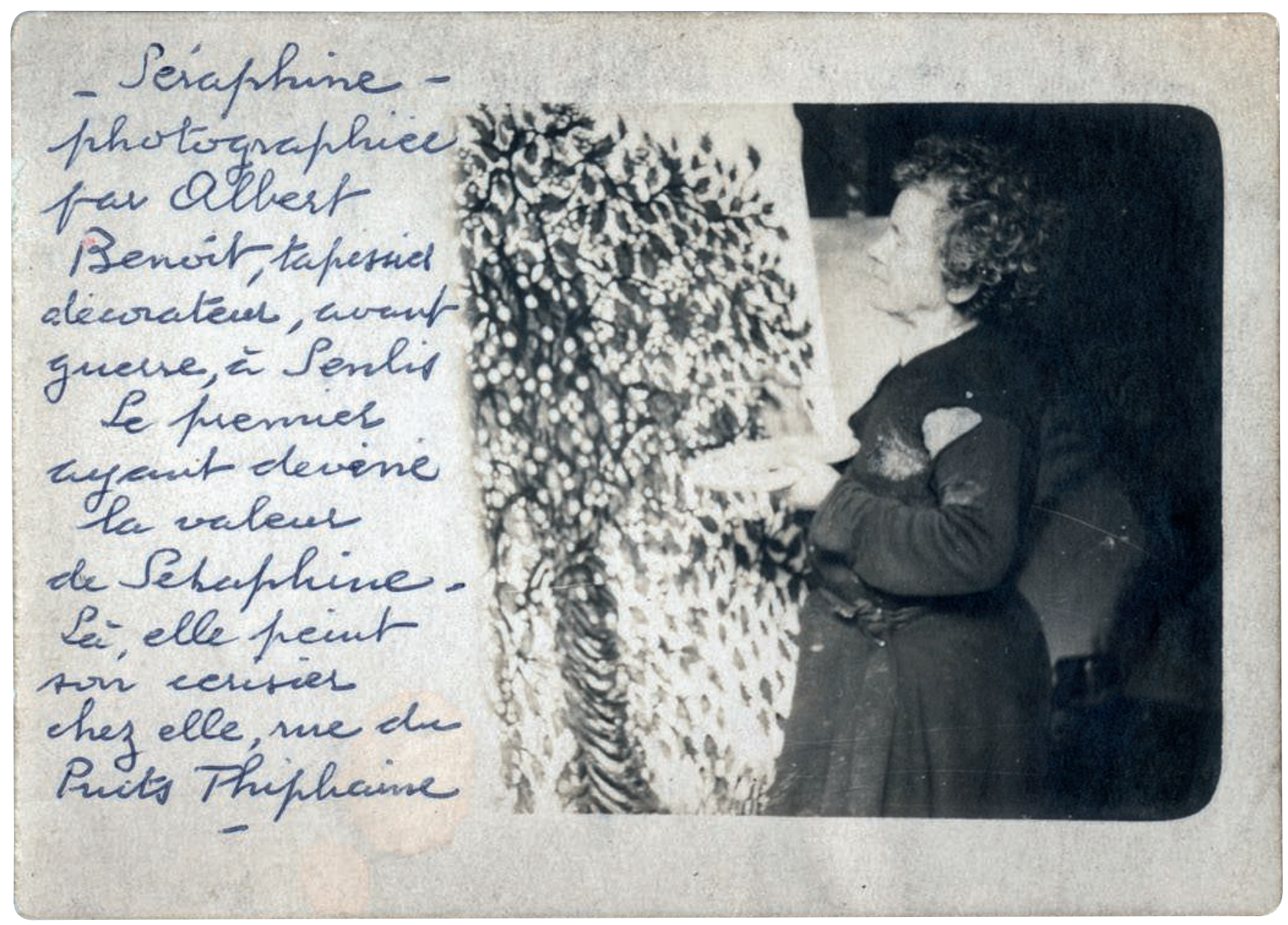

fig. 8

Séraphine Louis peignant, photographiée par Albert Benoît vers 1927-1928. DR. (c) Musées de Senlis. 


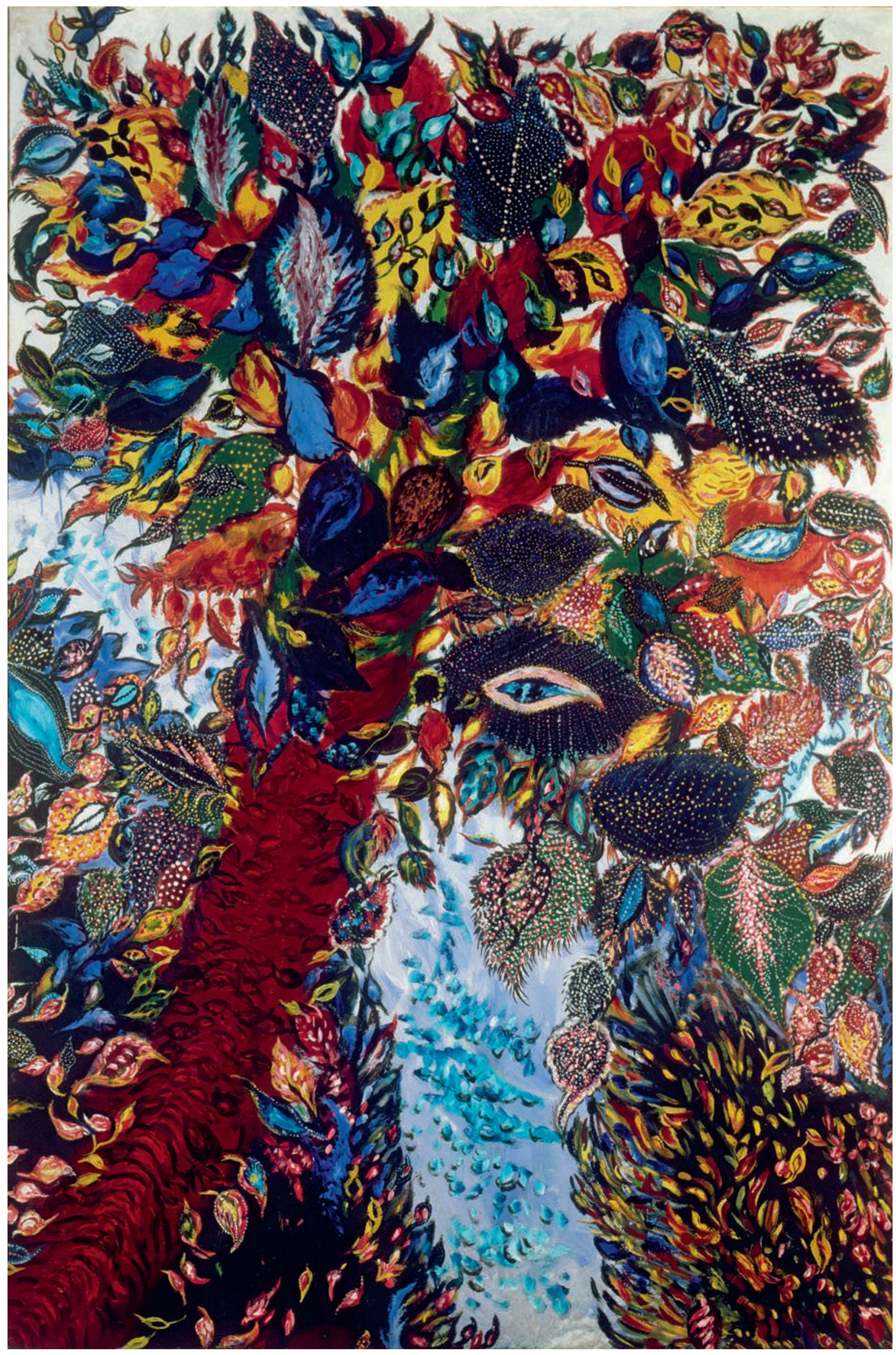


dans les trois séquences que nous avons détaillées. Non qu'il s'agisse de restaurer une croyance épuisée par le biais d'une narration invitant le spectateur à un pacte de véridicité. Mais plutôt de faire de cette version dégradée d'une vénérable théologie de l'image peinte une ressource pour insuffler à tout engagement créateur un peu de sacralité. Devant la presse, l'actrice et le réalisateur peuvent soutenir simultanément les deux points de vue puisqu'ils se sont eux-mêmes vécus comme «inspirés»:

Yolande Moreau: Je n'aime pas trop le mot «folie» à son propos. Aujourd'hui, on dirait qu'elle est une originale. C'est quelqu'un de mystique qui vit pour sa passion. Elle regarde la nature avec une sensibilité constante et peint parce que Dieu conduit sa main. [...] II y avait un climat très curieux: nous avions l'impression que Séraphine était présente auprès de nous...

Martin Provost: Souvent, il me semblait que nous étions guidés; je crois que cette réincarnation de Séraphine n'arrive pas par hasard... Cette histoire devait exister, et c'était le bon moment. 


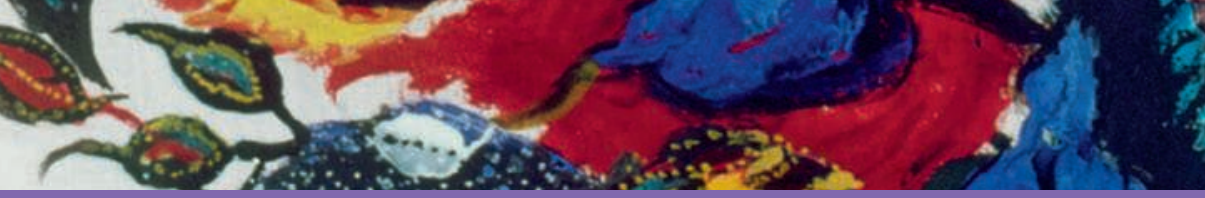

\section{Albera, François}

2008 « Cinéma et peinture, peinture et cinéma ", 1895. Mille huit cent quatre-vingt-quinze. Revue de l'Association française de recherche sur l'histoire $d u$ cinéma 54: 195-208.

\section{Aumont, Jacques}

2007 [1989] L'CEil interminable. Paris, La Différence (édition revue et augmentée).

\section{Bing, Henry}

1930 « Les limites de l'art populaire», Formes $2: 8$.

\section{Cassou, Jean}

1937 «Les maîtres populaires de la réalité», L'Art vivant 214: 201-203.

\section{Charuty, Giordana}

1997 Folie, mariage et mort. Pratiques chrétiennes de la folie en Europe occidentale. Paris, Seuil.

\section{Chevreffls Desbiolles, Yves}

2008 "Le critique d'art WaldemarGeorge. Les paradoxes d'un nonconformiste ", Archives juives 41: 101-117.

\section{Cloarec, Françoise}

2008 Séraphine. La vie rêvée de Séraphine de Senlis. Paris, Phébus.

\section{Costa, Antonio}

1991 Cinema e pittura.

Turin, Loescher.

2002 // cinema e le arti visive. Turin, Einaudi.

\section{Dagen, Philippe}

2008 Picasso. Paris, Hazan.

Delteil, Joseph

1930 «Bombois, peintre en peinture», Formes $2: 7$.

\section{Foucher, Jean-Pierre}

1968 Séraphine de Senlis. Paris, Les Éditions du Temps («L'œil du temps »).
Frangne, Pierre-Henry, Mouëllic, Gilles et Viart, Christophe (dir.)

2009 Filmer l'acte de création, Rennes, Presses Universitaires de Rennes ("Le spectaculaire»).

\section{Gallot, H.M.}

1957 «Séraphine, bouquetière "sans rivale" des fleurs maudites de l'instinct », L'Information artistique 40.

1968 Séraphine, 1864-1942, catalogue d'exposition. Paris, Galerie d'art de tradition populaire: 61-75.

\section{Gautherie-Kampka, Annette}

1995 Les Allemands du Dôme. La colonie allemande de Montparnasse dans les années 1903-1914, Berne, Berlin, Francfort, New York, Paris et Vienne, Peter Lang.

\section{Ginzburg, Carlo}

2013 Peur révérence terreur. Quatre essais d'iconographie politique. Dijon, Les presses du réel ("Oeuvres en sociétés")

\section{Goldwater, Robert}

1986 [1938] Primitivism in Modern Art. Cambridge et Londres, Harvard University Press.

\section{Gunther, Thomas}

Michael, Amanieux Françoise et de Labarthe, Marie-Christine

1989 La Réalité intérieure. Séraphine et quelques peintres autodidactes. Donations de W. et A.M. Uhde aux musées français. Senlis, Association de la sauvegarde de Senlis.

\section{Kahnweiler, Daniel-Henry}

1990 [1946] Juan Gris, sa vie,

son œuvre, ses écrits. Paris,

Gallimard («Folio »).

\section{Kandinsky, Wassily}

1974 Regards sur le passé et autres textes 1912-1922, Jean-Paul Bouillon (éd.). Paris, Hermann.
Körner, Hans et Wilkens, Manja

2009 Séraphine Louis 1864-1942.

Biographie/Werkverzeichnis.

Biographie/catalogue raisonné.

Berlin, Reimer-Benteli

\section{Labrusse, Rémi}

2009 « Délires anthropologiques. Joseph Strzygowski face à Aloïs Riegl», Histoire de l'art et anthropologie. Paris, INHA/musée du quai Branly («Les actes»); actesbranly.revues.org/268.

\section{Leblanc, Henri}

1972 Souvenirs d'enfance et de jeunesse, manuscrit. Senlis, bibliothèque du musée d'Art et d'Archéologie.

\section{Ligeia}

2008 «Peinture et cinéma. Picturalité de l'image filmée: de la toile à l'écran », nºs 77-80.

\section{Ortas-Peretti,}

\section{Marie-Amélie}

1965 Séraphine, peintre aliénée, thèse pour le doctorat en médecine, Paris.

\section{Provost, Martin}

s. d. Séraphine (d'après la vie de Séraphine de Senlis 1864-1942). Paris, TS Production.

\section{Quignard, Pascal}

2005 [1991] Georges de la Tour.

Paris, Galilée («Lignes fictives»).

\section{Recht, Roland}

2002-2003 "L'histoire de l'histoire de l'art en France (2) ", www.collegede-france.fr/media/roland-recht/

UPL56820_rrecht.pdf.

\section{Thivat, Patricia-Laure}

(dir.)

2011 Biographies de peintres

à l'écran. Rennes, Presses

Universitaires de Rennes

(«Le spectaculaire »).

\section{Uhde, Wilhelm}

1899 Am Grabe der Mediceer. Florentiner Briefe über deutsche Kultur. Dresde, Reißner.

1928 Picasso et la tradition française. Notes sur la peinture actuelle. Paris, Éditions des Quatre Chemins.

1929 «Défense de l'art populaire », Jazz 12: 537-539.

\section{1 «Séraphine ou la peinture} révélée ", Formes. Revue internationale des arts plastiques 17: 115-117.

\section{Cinq Maîtres primitifs} (Rousseau, Vivin, Bombois, Bauchant, Séraphine). Paris, Librairie Palmes-Philippe Daudy.

2002 De Bismarck à Picasso. Paris, Éditions du Linteau.

2008 Henri Rousseau, Séraphine de Senlis. Paris, Éditions du Linteau.

\section{Vircondelet, Alain}

1986 Séraphine de Senlis. Paris, Albin Michel ("Une vie»).

2008 Séraphine, de la peinture à la folie. Paris, Albin Michel.

\section{Waldemar-George}

1931 «André Bauchant. Romanité ou populisme», Formes 2 : 25-26.

page 108 et ci-contre Séraphine Louis dite de Senlis, L'Arbre du paradis, vers 1929, détail. Senlis, musée d'Art et d'Archéologie. Don d'Anne-Marie Unde, 1948. Dépôt du Centre Pompidou, 1989. @ Centre Pompidou, MNAM-CCI, Dist. RMN-Grand Palais/ Jacqueline Hyde. 


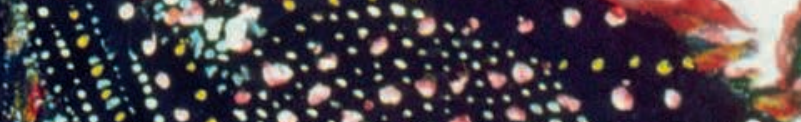

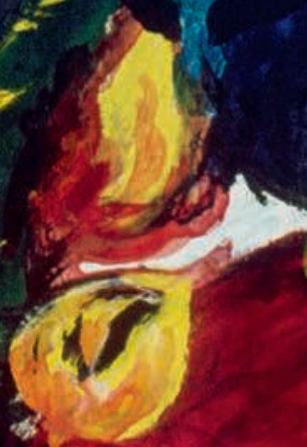

